\title{
Electronic cigarettes disrupt lung lipid homeostasis and innate immunity independent of nicotine
}

\author{
Matthew C. Madison, ${ }^{1,2}$ Cameron T. Landers, ${ }^{1,2}$ Bon-Hee Gu, ${ }^{3}$ Cheng-Yen Chang, ${ }^{1,2}$ Hui-Ying Tung, ${ }^{3}$ Ran You, ${ }^{3}$ Monica J. Hong, ${ }^{1,3}$ \\ Nima Baghaei, ${ }^{1}$ Li-Zhen Song, ${ }^{1}$ Paul Porter, ${ }^{3}$ Nagireddy Putluri, ${ }^{4}$ Ramiro Salas, ${ }^{5}$ Brian E. Gilbert, ${ }^{6}$ llya Levental, \\ Matthew J. Campen, ${ }^{8}$ David B. Corry, ${ }^{1,2,3,9,10}$ and Farrah Kheradmand ${ }^{1,2,3,9,10}$ \\ ${ }^{1}$ Department of Medicine, ${ }^{2}$ Interdepartmental Program in Translational Biology and Molecular Medicine, ${ }^{3}$ Department of Pathology and Immunology, ${ }^{4}$ Department of Molecular and Cell Biology, ${ }^{5}$ Department \\ of Psychiatry, and ${ }^{6}$ Department of Virology and Microbiology, Baylor College of Medicine, Houston, Texas, USA. D. Department of Integrative Biology and Molecular Pharmacology, University of Texas Health \\ Science Center, Houston, Texas, USA. ${ }^{8}$ Department of Pharmaceutical Sciences, College of Pharmacy, University of New Mexico, Albuquerque, New Mexico, USA. ${ }^{9}$ Biology of Inflammation Center, Baylor \\ College of Medicine, Houston, Texas, USA. ${ }^{10}$ Center for Translational Research onInflammatory Diseases (CTRID), Michael E. DeBakey VA Medical Center, Houston, Texas, USA.
}

\begin{abstract}
Electronic nicotine delivery systems (ENDS) or e-cigarettes have emerged as a popular recreational tool among adolescents and adults. Although the use of ENDS is often promoted as a safer alternative to conventional cigarettes, few comprehensive studies have assessed the long-term effects of vaporized nicotine and its associated solvents, propylene glycol (PC) and vegetable glycerin (VC). Here, we show that compared with smoke exposure, mice receiving ENDS vapor for 4 months failed to develop pulmonary inflammation or emphysema. However, ENDS exposure, independent of nicotine, altered lung lipid homeostasis in alveolar macrophages and epithelial cells. Comprehensive lipidomic and structural analyses of the lungs revealed aberrant phospholipids in alveolar macrophages and increased surfactant-associated phospholipids in the airway. In addition to ENDS-induced lipid deposition, chronic ENDS vapor exposure downregulated innate immunity against viral pathogens in resident macrophages. Moreover, independent of nicotine, ENDS-exposed mice infected with influenza demonstrated enhanced lung inflammation and tissue damage. Together, our findings reveal that chronic e-cigarette vapor aberrantly alters the physiology of lung epithelial cells and resident immune cells and promotes poor response to infectious challenge. Notably, alterations in lipid homeostasis and immune impairment are independent of nicotine, thereby warranting more extensive investigations of the vehicle solvents used in e-cigarettes.
\end{abstract}

\section{Introduction}

The electronic nicotine delivery system (ENDS), or e-cigarette, entered the US market in the mid-2000s and rapidly gained popularity among tobacco smokers and never-smokers (1). Currently, an estimated 10 million US adults and over 3 million high school age adolescents are active ENDS users $(2,3)$. Notably, ENDS have become the most commonly consumed tobacco substitute in the adolescent population, fueling concerns over the health-related consequences of ENDS exposure (4). A large contributor to the public appeal of ENDS among both adults and adolescents is the effective delivery of nicotine in the form of a vapor composed of the vehicle solvents propylene glycol (PG) and vegetable glycerin (VG), thereby circumventing the need for tobacco combustion. The popularity of ENDS has been further augmented by the additional flavorings that can be added to ENDS solutions. Over 7,000 flavors are available to accompany ENDS devices currently, which add essences of fruit or candy to enhance the experience of "vaping" (5).

Related Commentary: p. 4077

Conflict of interest: The authors have declared that no conflict of interest exists. Copyright: (c) 2019, American Society for Clinical Investigation.

Submitted: March 6, 2019; Accepted: July 23, 2019; Published: September 4, 2019

Reference information: J Clin Invest. 2019;129(10):4290-4304.

https://doi.org/10.1172/JCl128531.
ENDS-related studies often examine their addictive properties and the enticing advertising campaigns used by e-cigarette companies, which target impressionable youth (6). Specifically, studies have found that early ENDS use in teenagers may increase the risk for tobacco smoking later in life as they exhibit increased intention to smoke traditional cigarettes compared with those who have never used ENDS $(7,8)$. Moreover, toxicological studies have highlighted the potential health concerns of ENDS by revealing detectable levels of both heavy metals and carbonyls, such as formaldehyde and acrolein, in the vapor produced by some of the devices (9-11). Further, terms such as "popcorn lung" have become synonymous with ENDS among their opponents for the deleterious diacetyls detected in ENDS-associated flavorings (12). In contrast, other reports have advocated the safety of the products over traditional tobacco cigarettes $(13,14)$, thus emphasizing the unmet need to better understand the health-related consequences of exposure to e-cigarette components.

Multiple case reports have described atypical types of pneumonia in ENDS users $(15,16)$. A shared feature in these reports is the presence of lipid-laden macrophages found in the bronchoalveolar lavage (BAL) fluid from individuals with ENDS-associated pneumonia $(15,16)$. Despite this commonality, the physiological importance of lipid accumulation and whether this phenomenon can be recapitulated in experimental systems remain unknown. Lipid metabolism is critical for proper cellular function in all tis- 
sues. Pulmonary surfactant and lipid homeostasis in the distal airways are essential for adequate gas exchange and innate immune function (17). Pulmonary surfactant, a complex mixture of lipids and proteins produced exclusively by alveolar type II pneumocytes (ATIIs), forms a critical part of the alveolar-lining fluid (18). Surfactant reduces alveolar surface tension, thereby preventing lung atelectasis, ventilation perfusion mismatch, and hypoxemia (19). Surfactant protein A (SP-A) and surfactant protein D (SP-D), 2 components of the surfactant complex, play essential roles in innate immune defense by facilitating microbial opsonization and clearance (20). To maintain the integrity of the surfactant complex, alveolar macrophages catabolize altered or oxidized surfactant in the distal liquid-air interface, which is critical for responses to inhaled pathogens (21). Impairment of macrophage metabolism of pulmonary surfactant leads to accumulation of intracellular lipid and deposition of excess lipid in the alveolar space (22).

Notwithstanding the immunomodulatory roles of nicotine and its implications in cell proliferation and tumorigenesis $(23,24)$, little is known about how the constituents used in ENDS, namely PG and VG, affect the respiratory tract and its local immune functions. The Food and Drug Administration (FDA) recognizes these compounds as generally safe for use as direct food additives (25); however, whether and how these ENDS components exert significant effects on lung lipid homeostasis and innate immunity during long-term exposure remain unclear.

In this report, we used a murine model of chronic inhalation exposure to assess the effect of conventional tobacco smoke and components of ENDS on lung cellular function. Our model enabled a direct comparison between ENDS vapor and conventional smoke to examine their respective contributions to inflammation and lung cellular function. We show that the effect of chronic exposure to ENDS vapor is distinct from tobacco smoke, uniquely altering critical lipid-associated metabolic and immunological processes in the distal airway. Thus, our study findings sound an alarm for the potential harm of ENDS-associated products and could help provide new guidelines regarding their safety.

\section{Results}

Chronic smoke but not ENDS exposure induces lung inflammation and emphysema. We and others have shown that chronic cigarette smoke exposure activates innate and acquired immune cells in the lung and causes emphysema (26). To determine if ENDS exposure leads to similar outcomes, we first compared the lung-specific immunological effects of chronic ENDS exposure with exposure to conventional cigarette smoke. Compared with 4 months of cigarette smoke, C57BL/6J mice exposed to the same amount of nicotine delivered via ENDS (ENDS-nicotine) and those receiving ENDS without nicotine (ENDS-vehicle) showed no airway inflammation (Figure 1A). As expected, mice exposed to chronic cigarette smoke developed emphysema marked by an increase in lung volume, loss of the alveolar septa, and increased elastolytic enzymes (Figure 1, B-D). In contrast, histological assessment of the lungs in ENDS-exposed groups showed no evidence of tissue destruction when compared with the smoke-exposed group (Figure 1B); lung volume measurements with micro-computed tomography (microCT) revealed an increased lung volume in smoke-exposed mice, but not ENDS-exposed mice (Figure 1C). Consistent- ly, matrix metalloproteinase 12 (Mmp12) expression was highly induced in smoke-exposed mice but remained unchanged in the Air-control and ENDS-exposed mice (Figure 1D).

We and others have previously demonstrated that cigarette smoke recruits proinflammatory $\mathrm{CD} 11 \mathrm{~b}^{+} \mathrm{CD} 11 \mathrm{c}^{+}$conventional dendritic cells (cDCs) to the lung, which are critical in the differentiation of $\mathrm{T}$ helper 17 (Th17) cell responses in experimental emphysema (27). Therefore, we next sought to determine whether long-term ENDS exposure could induce cytokines that promote recruitment of $\mathrm{cDCs}$ and induction of Th17 cells. We found that chronic smoke increased IL-6, TNF- $\alpha$, and IL-17A concentrations in the lung, whereas ENDS exposure (with or without nicotine) did not increase any of the same inflammatory cytokines (Figure 1E). Further, flow cytometric analysis of lung tissue homogenate revealed increased recruitment of both Th17 cells and CD11b ${ }^{+}$ cDCs in response to smoke but not in the ENDS-exposed mice (Figure 1, F-I). To better understand the immunomodulatory consequences of ENDS exposure, we next evaluated lung cytokine and chemokine profiles from mice exposed to ENDS (with and without nicotine) and Air and found similar lung cytokine profiles among the 3 groups of Air-exposed and ENDS-exposed mice (Supplemental Figure 1A; supplemental material available online with this article; https://doi.org/10.1172/JCI128531DS1). Cytokines associated with Th2, Th1, or regulatory T cells (Tregs) in the lung homogenates were not significantly different in ENDS-exposed groups when compared to Air-exposed controls (Supplemental Figure 1A). Further, no distinct induction of chemokines was observed in the airways of END-exposed mice (with or without nicotine) (Supplemental Figure 1B). Together, these data suggest that chronic ENDS exposure in C57BL/6J mice does not promote notable inflammatory responses in the lung and does not induce emphysema.

ENDS exposure alters lipids in lung macrophages and ATIIs. Mice exposed to ENDS failed to exhibit a distinct inflammatory signature; however, we found a unique morphological feature in alveolar macrophages isolated from the BAL fluid of mice exposed to ENDS when compared with the Smoke or Air control groups (Figure 2A). Notably, interrogation of multiple pathways associated with autophagy (Bcln1, Atg7, Atg14) or proapoptotic (Bax, Bad) genes failed to reveal any significant upregulation of relevant genes (Supplemental Figure 2, A and B). Further, lactate dehydrogenase (LDH) concentrations in the BAL, a marker of cytotoxicity and cell death, were not increased in the ENDS-exposed groups (Supplemental Figure 2C). We next examined whether the observed cytoplasmic inclusions in macrophages contained lipids. Oil Red O staining of ENDS-exposed macrophages showed increased lipid accumulation independent of nicotine, a feature not observed in the Air- or Smoke-exposed groups (Figure 2B). Quantification of lipid in alveolar macrophages demonstrated a significant increase among both ENDS-exposed groups when compared with Air controls (Supplemental Figure 3). To determine whether the observed lipid accumulation was derived from inhaled vaporized glycerol present in ENDS, we next quantified the VG (glycerol) content in the BAL cellular fractions and found that ENDS-exposed groups did not exhibit an increased concentration of intracellular glycerol (Supplemental Figure 4), indicating that the accumulated lipid might be arising through an endogenous, rather than exogenous, source. 
A

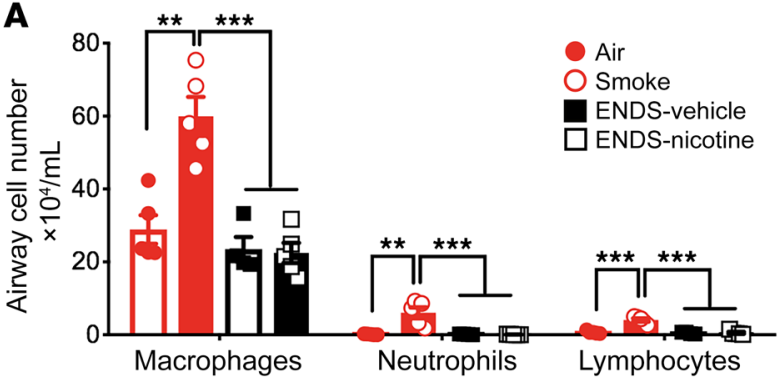

c

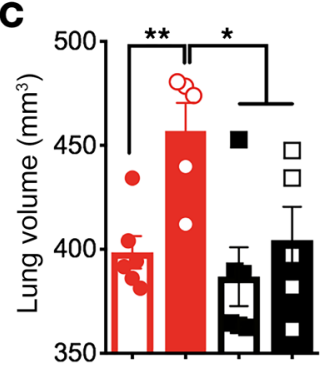

D $\quad M m p 12$

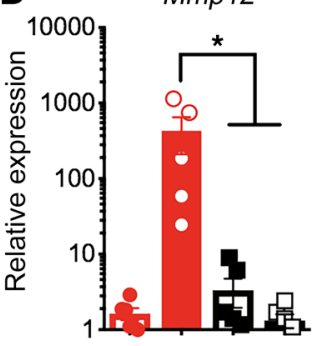

B
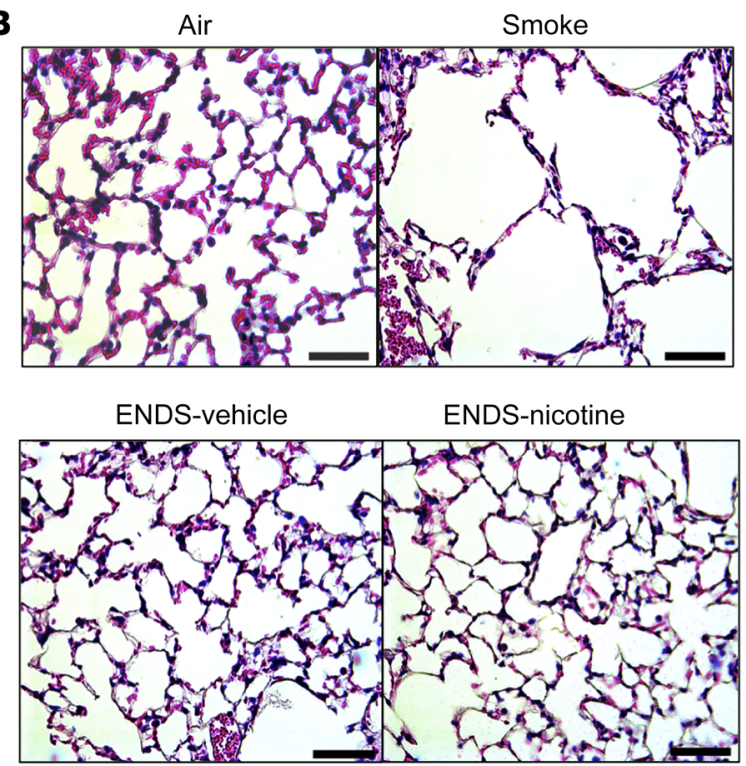

E

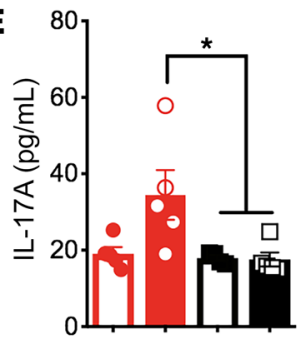

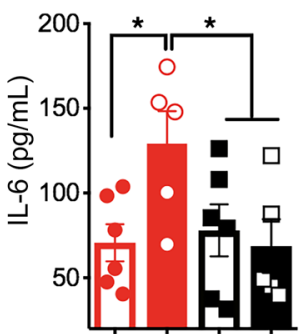

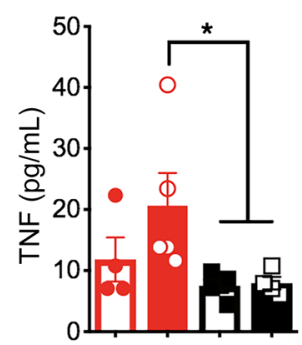

$\mathbf{F}$

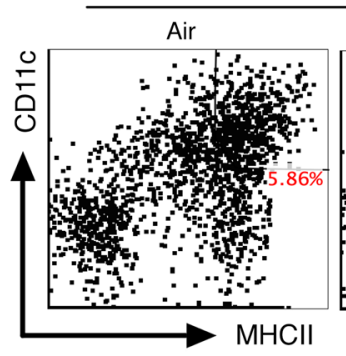

Lung CD11b+F4/80- Ly6g $^{-}$

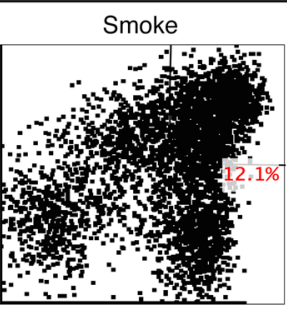

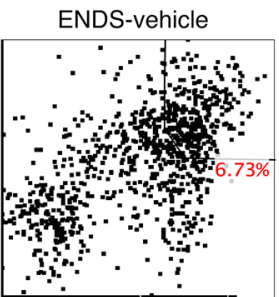

(3)

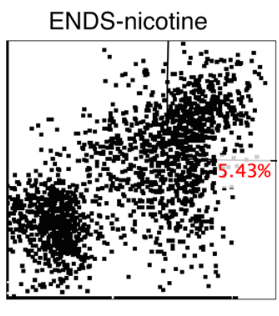

Lung $\mathrm{CD}^{+}{ }^{+} \mathrm{CD} 4^{+}$

H
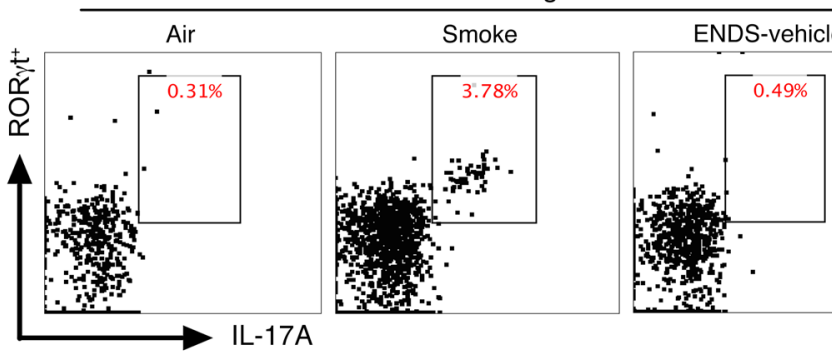

ENDS-nicotine

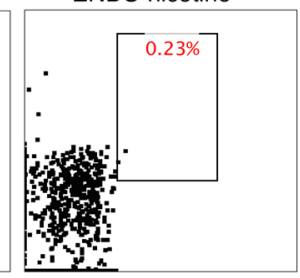

G Lung dendritic cell

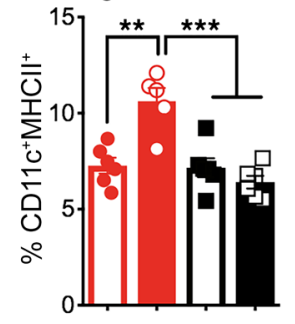

I

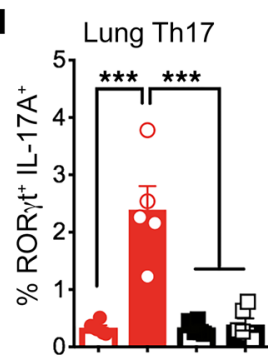

Figure 1. Four-month exposure to ENDS does not induce inflammation in the lung. Mice were exposed to room air (Air), cigarette smoke (Smoke), ENDS-vehicle vapor, or ENDS-nicotine vapor for 4 months and the immune profiles of the lung were quantified. (A) Differential BAL cell numbers for macrophages, neutrophils, and lymphocytes in the airway ( $n=5$ per group). (B) Histological analysis of lung tissue following 4-month exposure. Representative micrographs of H\&E staining. Scale bars: $50 \mu \mathrm{m}$. (C) MicroCT quantification of total lung volume ( $n=5$ or 6 per group). (D) BAL cell expression of RNA transcript for matrix metalloproteinase 12 by qPCR ( $n=5$ or 6 per group). (E) IL-17A, IL-6, and TNF- $\alpha$ concentrations from mouse lung homogenate measured

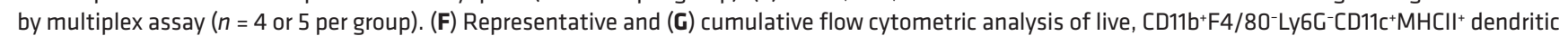
cells. Numbers in the upper-right corner indicate percentage positive cells for the markers ( $n=5$ or 6 per group). (H) Representative and (I) cumulative flow cytometric analysis of live, $C D 3^{+} C D 4^{+} R O R \gamma t^{+} I L-17 A^{+} T$ lymphocytes. Numbers in the upper-right corner indicate percentage positive cells for the markers ( $n=5$ or 6 per group). Significance was determined by Student's $t$ test or 1-way ANOVA with Bonferroni's correction for multiple comparisons. ${ }^{* * *} P<0.001$, ${ }^{* *} P<0.01,{ }^{*} P<0.05$. All data shown are representative of 4 or more independent 4-month experiments with $n=5$ or 6 . 


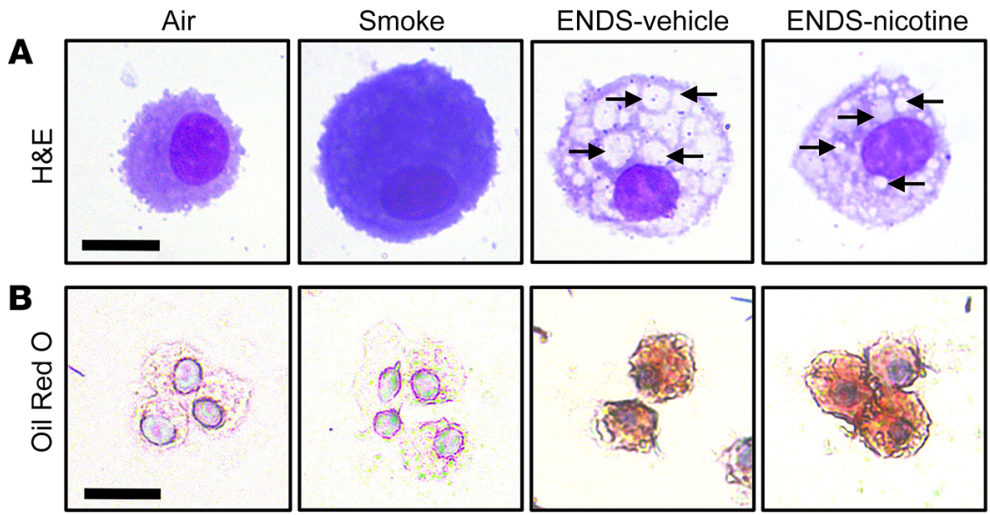

Figure 2. Lipids accumulate in alveolar macrophages in chronic ENDS exposure. Mice were exposed to Air, Smoke, ENDS-vehicle, or ENDS-nicotine for 4 months. Airway immune cells were then acquired by BAL and were cytocentrifuged onto glass slides. (A) Representative H\&E staining of the cytospin preparations reveals intracytoplasmic inclusions in the ENDS-vehicle and ENDS-nicotine groups (black arrows). Scale bar: $10 \mu \mathrm{m}$. (B) Representative Oil Red 0 staining of cytospin preparations reveals lipid accumulation in ENDS-vehicle and ENDS-nicotine groups. All data shown are representative of 3 or more independent 4-month experiments with $n=4$ or 5 per group. Scale bar: $25 \mu \mathrm{m}$.
We next used transmission electron microscopy (TEM) and found numerous lipid aggregates in the cytoplasm of macrophages isolated from ENDS-exposed mice (Figure 3, A and B). Furthermore, we found an increase in the number of lysosomes in ENDS-exposed macrophages (with or without nicotine), which we confirmed with immunohistochemical staining for lysosomalassociated membrane protein 1 (LAMP-1) (Figure 3B and Supplemental Figure 5). We next examined the lamellar bodies, the specialized secretory vesicles in ATIIs that facilitate the release of the lipid constituents of pulmonary surfactant into the alveolar airspace. Similarly, the lamellar bodies in the ENDS-exposed groups also demonstrated distinct morphological changes, when compared with the Air-exposed counterparts (Figure 3, C and D). Although the absolute number of lamellar bodies was unaltered, the numbers of poorly organized, irregular lamellar bodies were increased in both ENDS-treated groups, suggesting an alteration in ATII surfactant homeostasis had occurred (Figure 3E). Collectively, these data indicate that ENDS vapor exposure disrupts lipid homeostasis in alveolar macrophages and ATIIs, warranting further systematic assessment of the airway's lipidome.

ENDS-exposed mice accumulate phospholipid in BAL cells. We next used an unbiased, mass spectrometry-based lipidomic approach to assess how ENDS vapor exposure disrupts the lipid landscape in the lungs. We have found that alveolar macrophages constitute over $98 \%$ of the BAL fluid cell composition in mice exposed to 4 months of ENDS-vehicle, ENDS-nicotine, or Air control (Figure 1A and Supplemental Figure 6, A and B). Therefore, we next examined the lipidomic changes in the BAL cells in the same exposed groups of mice. Independent of nicotine exposure, we found an increase in cellular phospholipid species in the ENDS-treated groups, including the phosphatidylcholine-, phosphatidylserine-, and phosphatidylethanolamine-based lipids, with an enrichment of disaturated phospholipids and cholesterol esters (Figure 4A). These findings were specific because our comprehensive analyses of other classes of lipids (e.g., triglycerides) did not show significant alterations (Supplemental Figure 7). The observed shifts in cellular phospholipids from the lipidomic analysis were further validated by quantification of total cellular phospholipids in BAL fluid cells isolated from a separate cohort of mice exposed to chronic ENDS. Intracellular phospholipids were significantly increased in the ENDS-nicotine-treated group. (Figure $4 \mathrm{~B})$. To determine whether ENDS exposure altered pathways associated with lipid maintenance and clearance, we next examined gene expression patterns of lipid-associated enzymes and transport proteins. We found that expression of Abca1, a transport molecule responsible for removal of excess intracellular cholesterol and phospholipid, was reduced in ENDS-exposed groups compared with Air controls (Supplemental Figure 8A). A similar trend was also observed in the expression of $A b c g 1$, a sterol efflux protein that works in conjunction with ABCA1 to maintain lipid balance in alveolar macrophages (Supplemental Figure 8A). Together, our findings suggest that ENDS-exposed, alveolar macrophages show defects in complex lipid processes in the airway, which may, in part, promote the accumulation of distinct lipid species.

Surfactant-associated lipid species are increased in ENDSexposed lungs. To acquire insight into the mechanisms responsible for altered intracellular lipid biosynthesis, we next examined the lipid profiles in the acellular compartment of the BAL fluid. This analysis revealed that ENDS-exposed groups have a distinct increase in phospholipid species with a concomitant decrease in neutral triglycerides (Figure 5A). The acellular fractions from ENDS-exposed mice further showed significant increases in saturated phospholipids with concurrent decreases in phospholipids bearing more double bonds (Figure 5, B and C). To ascertain whether the observed shifts in the lipid profiles of ENDS-exposed mice were related to an increase in pulmonary surfactant, we next identified and quantified lipid species that are most prominent in surfactant from the lipidomics data set: dipalmitoyl-phosphatidylcholine (DPPC), myristoyl-palmitoyl-phosphatidylcholine (MPPC), palmitoyl-stearoyl-phosphatidylcholine (PSPC), and palmitoyl-palmitoleoyl-phoshpatidylcholine (PPoPC) (Figure 5D). These phospholipids are estimated to constitute over $40 \%$ of the phospholipid compartment of the surfactant layer and primarily function to reduce alveolar surface tension $(28,29)$. We found that independent of nicotine, ENDS exposure significantly increased the concentration of several of these phospholipids, including MPPC and PSPC. Together, these findings show that chronic ENDS vapor exposure disturbs phospholipid homeostasis in the surfactant layer, resulting in a distinct increase in the alveolar phospholipid pool.

To further characterize the effect of ENDS vapor on the biosynthetic function of ATIIs, we assessed the expression of proteins that are critical for the assembly and transport of pulmonary surfactant. We found that $A b c a 3$, a type II-specific lipid transport protein that 
A

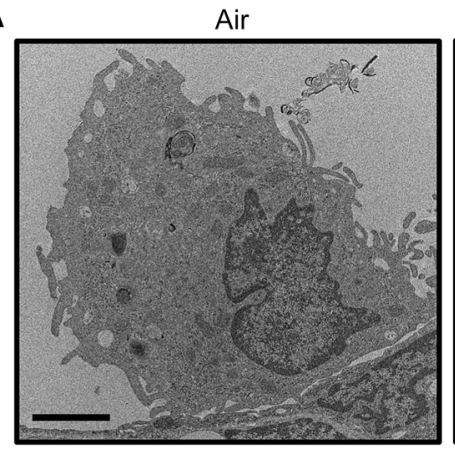

ENDS-vehicle

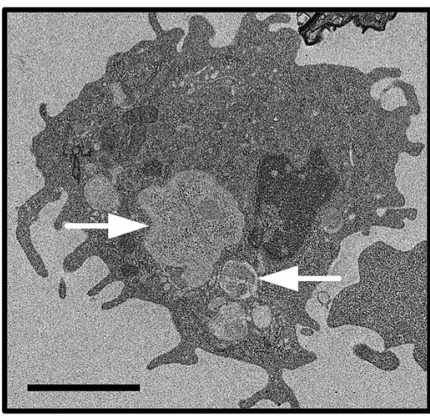

ENDS-nicotine

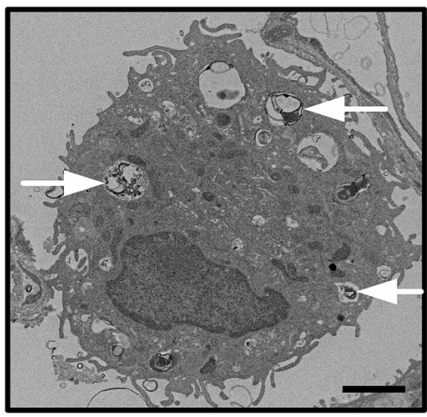

B

ENDS-vehicle

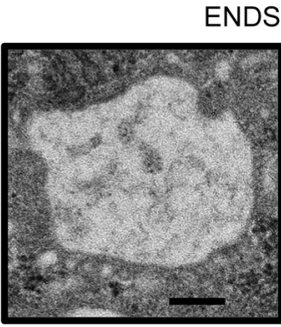

ENDS-nicotine
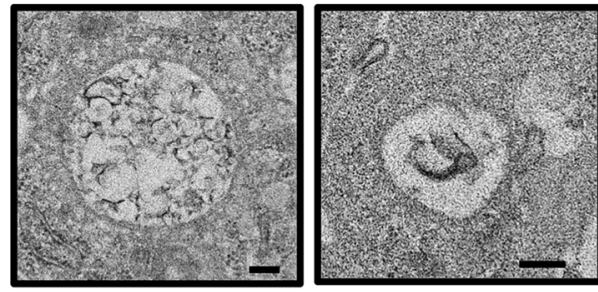

C

Air
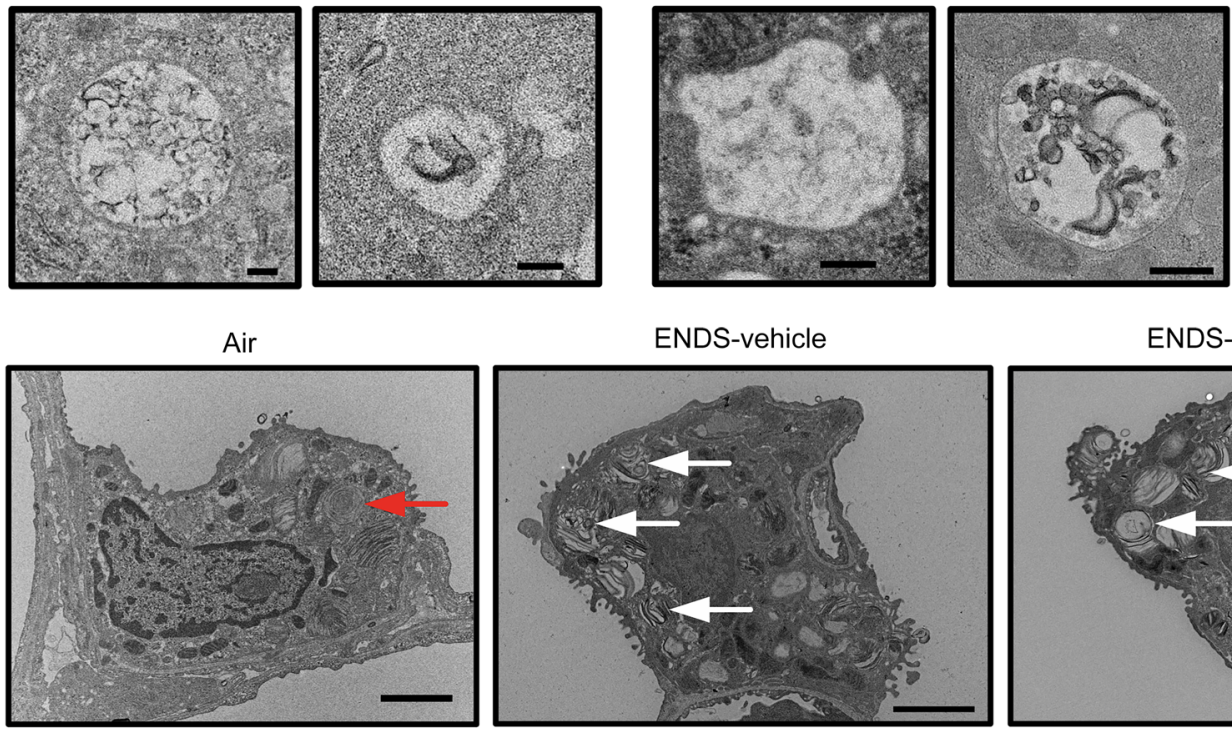

D

Air

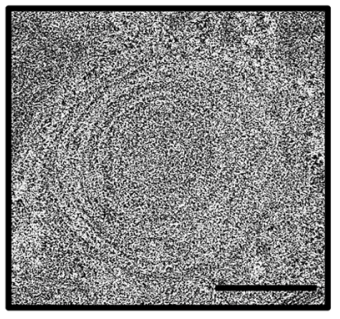

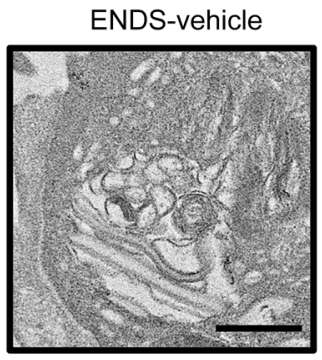
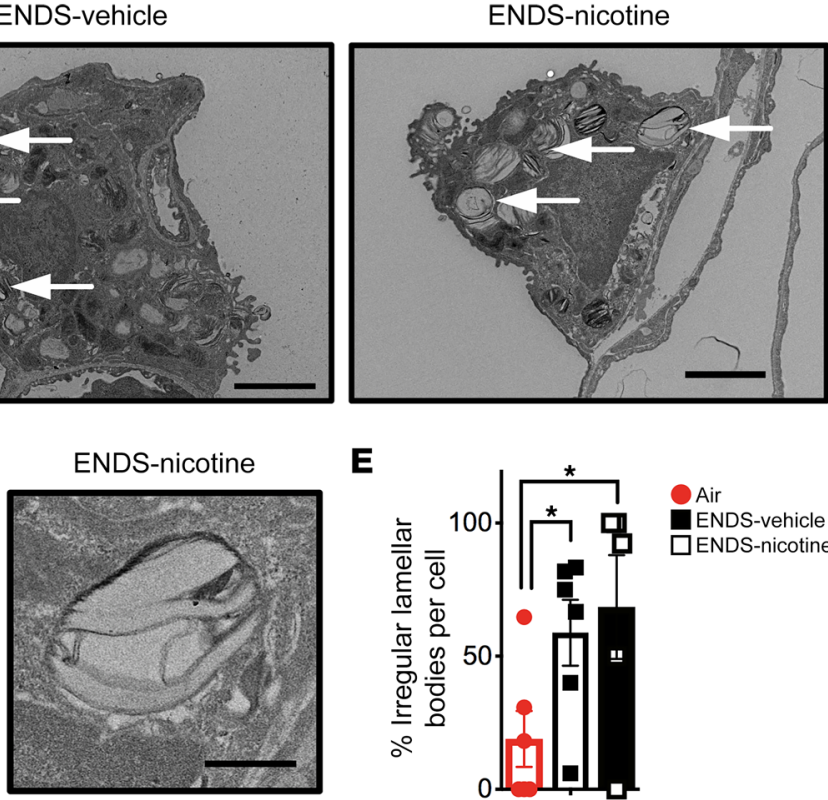

E

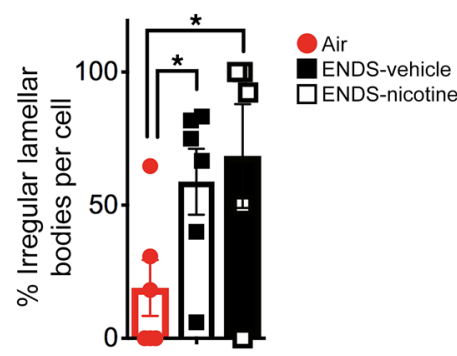

Figure 3. TEM imaging of lipid inclusions in alveolar macrophage and alveolar type II pneumocytes. Following 4 months of exposure, lungs from Air, ENDS-vehicle, and ENDS-nicotine groups were fixed and processed for electron microscopic analysis. (A) Representative micrographs of alveolar macrophages demonstrating lipid inclusions and increased presence of lysosomal compartments in ENDS-vehicle and ENDS-nicotine groups (white arrows). Scale bars: $2000 \mathrm{~nm} .80 \mathrm{kV}$ high voltage. (B) Higher magnification of the lipid inclusions (right) and lysosomes (left) observed in ENDS-vehicle and ENDS-nicotine groups. Scale bars: $200 \mathrm{~nm} .80 \mathrm{kV}$ high voltage. (C) Representative micrographs of alveolar type II pneumocytes (ATIls) demonstrating normal, uniform lamellar bodies in AIR-exposed mice (red arrow) and atypical lamellar body structures in ENDS-vehicle and ENDS-nicotine groups (white arrows). Scale bars: $2000 \mathrm{~nm} .80 \mathrm{kV}$ high voltage. (D) Higher magnification of the representative lamellar bodies observed in AIR, ENDS-vehicle, and ENDS-nicotine groups. Scale bars: $500 \mathrm{~nm} .80 \mathrm{kV}$ high voltage. (E) Blinded quantification of atypical lamellar bodies observed within ATIls. The quantified results are expressed as the percentage of atypical lamellar bodies per total lamellar body count in each cell (mean \pm SEM). $n=5$ or 6 per group. Each data point represents a single ATII, all of which were located and imaged by scanning 3 or more independent mounted grids per experimental group. Significance was determined by Student's $t$ test. ${ }^{*} P<0.05$.

aids in the building of lamellar bodies, was upregulated in response to ENDS-vehicle, with ENDS-nicotine demonstrating an additive effect (Supplemental Figure 8B). We also found that expression of Pcyt1a and Lpcat1, genes for the phosphatidylcholine-synthesizing enzymes CTP:phosphacholine cytidylyltransferase and lysophos- phatidylcholine acyltransferase 1, were upregulated in ENDSnicotine-exposed groups (Supplemental Figure 8B).

The cytokine granulocyte macrophage colony-stimulating factor (GM-CSF) plays an important role in macrophage physiology and lipid metabolism (30). Therefore, we next sought to deter- 


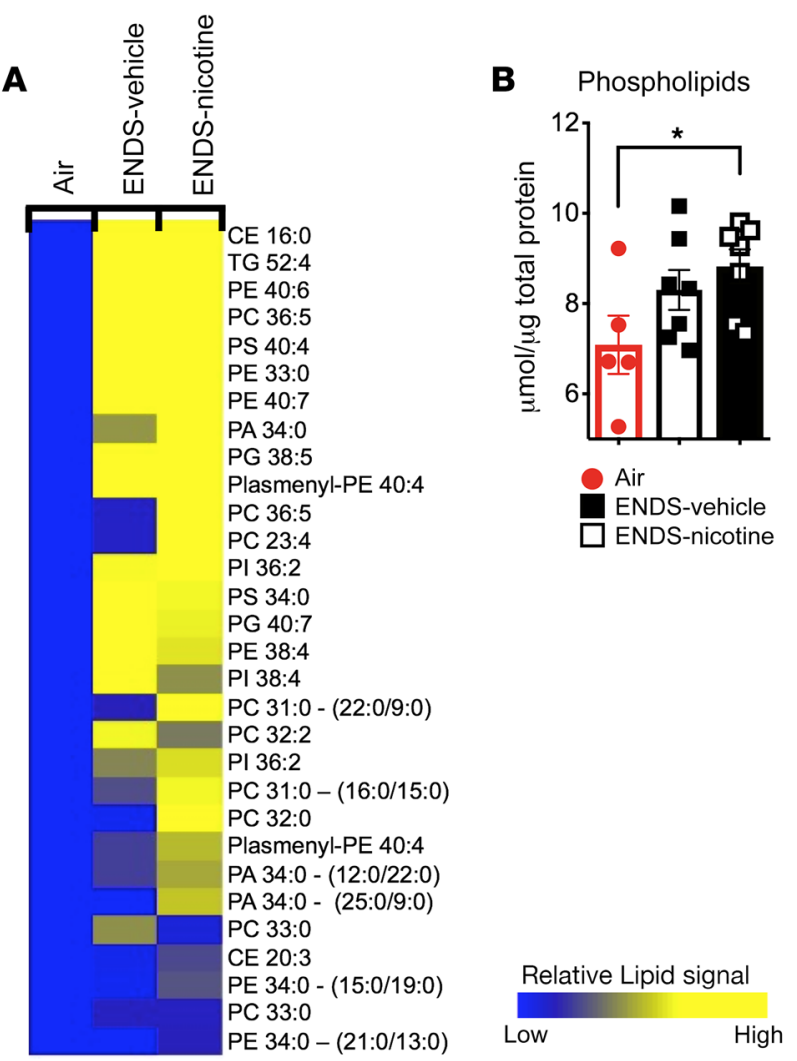

mine if expression of GM-CSF is altered in mice with abnormal lipid homeostasis in response to ENDS exposure. Interestingly, we found no significant alteration in GM-CSF protein levels from whole lung homogenate, GM-CSF receptor (Csf $2 r a$ ), or PU.1 expression, a downstream transcription factor that regulates expression of proteins necessary for surfactant maintenance and catabolism in BAL fluid cells (Supplemental Figure 9, A-C). Together with the disruption in lipid homeostasis, these data suggest that chronic ENDS exposure alters the surfactant-related lipid output in the terminal airway and disrupts key metabolic pathways by which alveolar macrophages process and catabolize the lipids at the alveoli's air-liquid interface in a manner that is independent of GM-CSF.

ENDS exposure alters innate immune functions of ATIIs and lung macrophages. Although ENDS exposure resulted in increased abundance of lipid species associated with the surfactant proteins, mice receiving ENDS showed significantly reduced SP-D concentrations in the BAL fluid (Figure 6A). Furthermore, gene expression analysis revealed that mRNA transcripts for 2 opsonins, Sfptd and Sfpta, were significantly reduced in the lung homogenates from ENDS-exposed mice when compared with Air controls (Figure 6B). Notably, these findings were specific because no significant changes were found in Sfptb and Sfptc (Figure 6C). Together, this demonstrates that ENDS vapor exposure impairs production of an essential class of opsonins in the distal airway.

Using the same exposure protocol, we found that lipids are deposited in lung macrophages as early as 2 weeks (Supplemental Figure 10). Therefore, to examine the early immunological effects of ENDS vapor exposure on lung macrophages, we isolated $\mathrm{F} 4 / 80^{+}$macrophages from the whole lung tissue of mice after 1
Figure 4. ENDS exposure independent of nicotine increases phospholipids in BAL cells. (A) Heatmap demonstrating the upregulated phospholipids in ENDS-vehicle and ENDS-nicotine groups (fold change > 1.5) from the lipidomic analysis conducted on the BAL cells. Heatmap values represent averages from the 3 pooled samples per group. Changes shown are relative to the Air controls. (B) Quantification of total intracellular phospholipid content in pelleted BAL cells. $n=5-7$ per group. The quantified results are expressed as means \pm SEM. Significance was determined by Student's $t$ test and corrected for multiple comparisons. ${ }^{*} P<0.05$. CE, cholesterol ester; TG, triglyceride; PE, phosphatidylethanolamine; PC, phosphatidylcholine; PS, phosphatidylserine; $\mathrm{PI}$, phosphatidylinositol; PA, phosphatidic acid; PG, phosphatidylglycerol.

month of exposure. We found similar cell viability and expression of M2 polarization markers (e.g., Arg1) in ENDS-exposed F4/80+ lung macrophages when compared with Air-exposed controls (Figure 7, A and B). In contrast, lung macrophages showed significantly reduced M1-associated markers, including Nos2 (Figure 7C), proinflammatory cytokines (Il1b and Tnfa) (Figure 7D), B7 costimulatory molecules ( $C d 80$ and $C d 86$ ) (Figure 7E), and $T \operatorname{lr} 7$, a pattern-recognition receptor that recognizes single-stranded RNA elements from viral species (Figure 7F).

To determine the functional significance of reduced M1-associated cytokines and molecules, we next used polyinosinic:polycytidylic acid (poly I:C), a synthetic analog of viral dsRNA, to stimulate macrophages isolated from the lungs of ENDS-exposed mice. Consistently, in response to poly I:C, the macrophages from ENDSexposed groups showed reduced expression of interferon (IFN) response factor 7 (Irf7) (Figure 7G). IRF7 is a master transcription factor that governs type 1 IFN induction in response to viral stimuli, which is critical for rapid antiviral immunity (31). This reduction, along with the decrease in M1-associated markers, strongly indicates that one potentially important physiological consequence of ENDS vapor exposure is an impaired response to inhaled viruses.

ENDS-exposed mice exhibit delayed immune responses to influenza. To further examine the translational relevance of reduction of M1-associated markers, impaired macrophage responses to poly I:C, and surfactant dysregulation in mice exposed to ENDS vapor, we next assessed defenses against acute viral infection. We have previously shown that mice exposed to chronic cigarette smoke, before sublethal infection with influenza A, fail to mount the appropriate antiviral responses and exhibit increased morbidity (32). In a similar manner, mice were exposed to Air (control), chronic ENDS-vehicle (PG/VG), or ENDS-nicotine for 3 months and were subsequently challenged with the influenza A virus (45 $\mathrm{TCID}_{50} /$ mouse). Mice receiving ENDS responded poorly by day 10 following infection, and the ENDS-vehicle group demonstrated a significant increase in mortality when compared with Air controls (Figure 8A). To better understand the effects of ENDS on the recovery from acute infection, we next exposed mice to ENDS vapor for 3 months and infected with a sublethal dose of influenza A (20 $\mathrm{TCID}_{50} /$ mouse). Compared with the Air group, ENDS-nicotineexposed mice showed significantly impaired responses to influenza A, as detected by augmented weight loss within 8 days following infection (Figure 8B). As expected, ENDS-exposed macrophages in the BAL showed the same lipid-filled morphology (Figure 8C). The Air-exposed control group showed resolution of inflammation and had regained some of the normal lung architecture at 
A Air ENDS-vehicle ENDS-nicotine

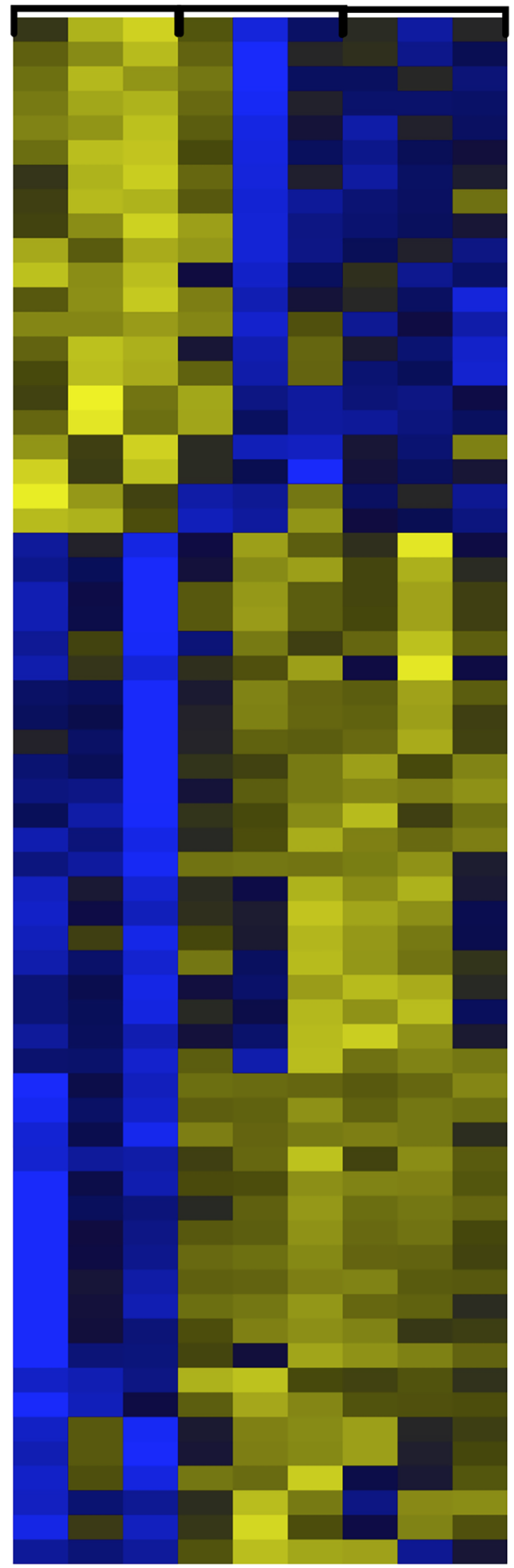

G (16:0/18:0/18:2) TG (14:0/16:1/18:1) TG (16:0/16:0/18:2)

TG (16:0/18:1/18:1)

TG (16:0/18:1/18:1)

TG (16:0/18:0/18:1)

TG (14:0/16:1/18:1)

TG (18:1/18:1/18:2)

TG (18:0/18:1/18:2)

TG (16:0/16:0/18:2)

TG (16:0/16:0/16:1)

TG (16:0/16:0/18:1)

TG (16:0/16:0/18:1)

IysoPC (20:4/0:0)

TG (16:0/16:0/16:1)

TG (16:1/16:1/18:1)

TG $(16: 0 / 16: 1 / 18: 2)$

TG (18:0/18:2/18:2)

ysoPC (12:0/0:0)

PE (16:1/20:4)

PE (16:0/18:1)

plasmenyl-PE (16:0/20:4)

PC (14:0/16:0)

PC (18:0/16:0)

PE (17:0/19:0)

PG (14:0/16:0)

plasmenyl-PE (16:0/22:4)

PI (2:0/22:1)

$P C(16: 0 / 16: 0)$

$P C$ (16:0/16:0)

PE (18:0/20:4)

$P C(14: 0 / 16: 0)$

PE (16:0/22:5)

PC (17:1/18:1)

PC (18:0/20:4)

$P C(8: 0 / 26: 0)$

PC (17:0/17:0)

PC (21:0/11:0)

PC (17:1/18:2)

plasmenyl-PE (16:0/22:4)

PC (7:0/24:0)

plasmenyl-PE (18:0/20:1)

PA (6:0/16:1)

PE (17:0/18:0)

MG (22:4/0:0/0:0)

MG (15:1/0:0/0:0)

PC (18:0/18:0)

PE (3:0/24:4)

MG (21:0/0:0/0:0)

PA (9:0/18:4)

PC (18:3/19:0)

SM $(15: 0 / 18: 1)$

$P C$ (17:0/22:6)

PC (17:0/22:6)

$P C(3: 0 / 13: 0)$

PC (14:1/24:4)

$\operatorname{MGDG}(2: 0 / 18: 1)$

SM (19:0/24:4)

PC (15:0/16:1)

$P C(15: 1 / 16: 0)$

$P C(16: 1 / 16: 1)$

PI (17:0/20:4)

PS (17:0/17:0)

PC (26:0/9:0)

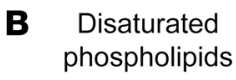

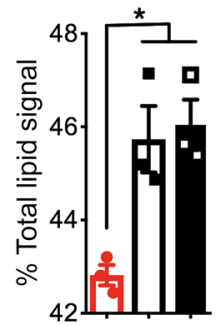

Air

ENDS-vehicle

ENDS-nicotine
C

Phospholipids: Phospholipids:

2-DB
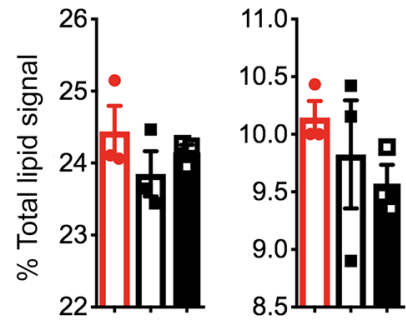

Phospholipids: Phospholipids:

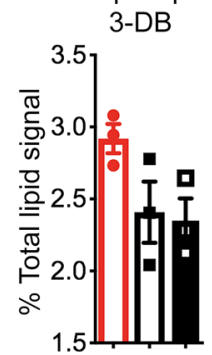

4-DB

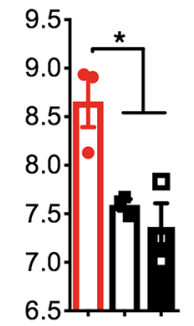

D

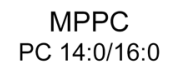

MPoPC
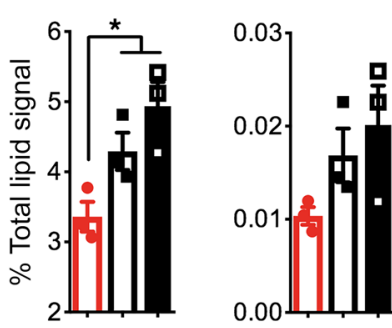

PC 18:0/16:0

\section{DPPC}
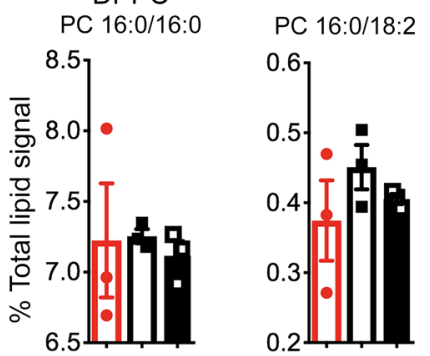

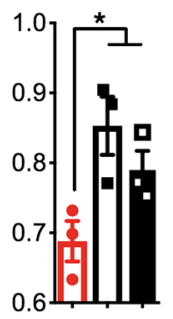

PC 16:0/16:1

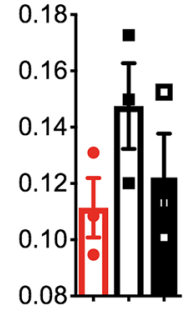

Figure 5. ENDS exposure independent of nicotine increases disaturated phospholipid pools in BAL fluid. (A) Heatmap depicting the upregulated phospholipid species in the ENDS-vehicle and ENDS-nicotine groups from the lipidomic analysis conducted on the BAL fluid. $n=3$ pooled samples per group. (B and C) Quantification of BAL fluid phospholipids based on saturation of the lipid acyl groups. $n=3$ per group. The quantified results are expressed as percentage of total lipid signal (mean \pm SEM). DB, double bond. (D) Quantification of known surfactant-associated species in BAL fluid. $n=3$ per group. The quantified results are expressed as percentage of total lipid signal (mean \pm SEM). Significance was determined by 1-way ANOVA with Bonferroni's correction for multiple comparisons. ${ }^{*} P<0.05$. CE, cholesterol ester; TC, triglyceride; PE, phosphatidylethanolamine; PC, phosphatidylcholine; PS, phosphatidylserine; PI, phosphatidylinositol; PA, phosphatidic acid; PG, phosphatidylglycerol; SM, sphingomyelin; MG, monoacylglycerol; MGDG, mono/diacylglycerol.

day 14; however, histological evaluation of the lung parenchyma in ENDS-exposed mice showed increased lung inflammatory cells and edema with significant distortion of the lung tissue and increased hemorrhage in ENDS-exposed mice when compared with the Air-exposed mice that were infected with flu (Figure 8, $\mathrm{D}$ and $\mathrm{E})$. Consistent with the increase in inflammatory infiltrates in the lungs, there was persistent IFN- $\gamma$ expression in the lungs of ENDS-nicotine-treated groups, and TNF- $\alpha$ in the ENDS-vehicle- 

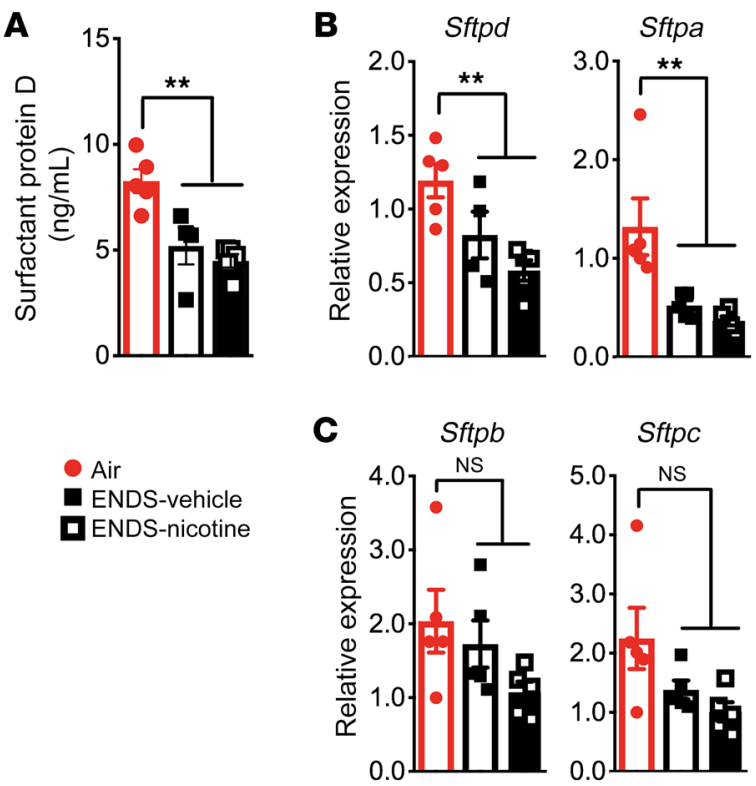

Figure 6. Four-month exposure to ENDS vapor reduces the expression of surfactant proteins. (A) Quantification of SP-D by ELISA from BAL fluid. $n=4$ or 5 per group. (B and $\mathbf{C}$ ) Relative gene expression of surfactantassociated proteins (SP-A, SP-B, SP-C, and SP-D) from whole lung homogenate. $n=4$ or 5 per group. The quantified results are expressed as means \pm SEM. Significance was determined by 1-way ANOVA with Bonferroni's correction for multiple comparisons. ${ }^{* *} P<0.01$. NS, not significant.

exposed mice (Figure $8 \mathrm{~F}$ ). We further found increases in total and HA-specific IgG titers in the lung homogenate of ENDS-exposed mice 14 days following viral infection (Figure 8, G and H). Together, our data demonstrate that exposure to ENDS vapor reduces innate immune responsiveness, and long-term exposure impairs the ability of mice to control pulmonary infection with influenza.

\section{Discussion}

We have uncovered a harmful effect of vaporized e-cigarette products in the alveolar-lining fluid and the lung, summarized in Figure 9. To our knowledge, this is the first report that demonstrates how chronic exposure to ENDS, independent of nicotine, alters lung lipoprotein biology in ATIIs and impairs the physiology of alveolar macrophages. Prior reports have shown that vaporized nicotine can disrupt immune cell functions, increase lung inflammation, and promote emphysema in response to short-term and long-term ENDS exposure (33-35). We show here that long-term exposure to ENDS vapor, which delivers nicotine in amounts equal to those of conventional cigarettes, did not result in excessive lung inflammation or emphysema. Despite these findings, mice exposed to ENDS and infected with the flu virus had significantly delayed immune responses to the infection and showed persistent lung inflammation when compared with the Air-exposed group. Further, the increase in morbidity and mortality was independent of nicotine, indicating that the harmful effects of chronic inhalation of ENDS vapor is due to the PG/VG vehicles, which are currently considered safe solvents (25).

An intricate and essential relationship exists between alveolar macrophages and the pulmonary surfactant that lines the distal airways (36). Gene expression profiling of resident alveolar macrophages is consistent with an enrichment of genes associated with lipid metabolism, thus highlighting their significant role in pulmonary surfactant homeostasis $(36,37)$. Our study demonstrates that chronic exposure to PG/VG uniquely promotes deposition of lipids in alveolar macrophages and disturbs the normal homeostatic process of pulmonary surfactant molecules and lipids in the lungs. Under homeostatic conditions, lung-resident macrophages play a crucial role in maintaining the composition and integrity of the surfactant layer by catabolizing (e.g., recycling) aggregated surfactant that has been oxidized or altered at the liquid-air interface (38). Furthermore, lung surfactant modulates the function of the innate immune cells of the distal airway, including alveolar macrophages. Particularly, surfactant-associated proteins maintain surfactant homeostasis, and play critical roles in the function of alveolar macrophages (39). Genetic ablation studies have revealed that the role of surfactant proteins goes far beyond innate immune function and highlight implications for critical roles in cellular metabolism and lipid processing. Specifically, SP-D-deficient mice display defects in lipid biogenesis and show accumulation of lipid-laden macrophages in the distal airway as well as marked increases in the phospholipid pools of surfactant (40).

ATIIs use SP-D to monitor the size and condition of the surfactant pool in the alveoli, indicating its function as a vital mediator of surfactant recycling and catabolism (41). Intriguingly, our model of ENDS exposure mimics several aspects of the phenotype observed in SP-D $\mathrm{D}^{-/-}$animals. Although surfactant-associated phospholipid pools increased in long-term ENDS vapor exposure, we found a significant decrease in the expression of the hydrophilic, surfactant-associated proteins SP-A and SP-D in the BAL fluid. The metabolic alteration in surfactant protein synthesis and the increase in surfactant-associated lipids may, in part, cause the disruption of alveolar macrophage function and immunological changes detected in mice exposed to chronic ENDS vapor. These findings reemphasize the important regulatory link between surfactant and macrophage physiology, which we reveal is disturbed in response to chronic exposure to the vaporized solvents. Additionally, since the ENDS-mediated effects are observed even in the absence of nicotine, it raises important health concerns regarding the solvents used in noncombustible inhaled products.

Biological membranes are critical tissue components that are responsive to both endogenous and exogenous stimuli (42). As a vital biological component of the airway fluid, pulmonary surfactant maintains the normal physiology of resident cells and is critical for proper gas exchange (43). The membranous surfactant structure can be perturbed by a number of molecules, which diminishes its surface tension-reducing arrangement and impairs lung function (43). For instance, excess cholesterol in the surfactant layer interrupts the organization and distribution of the lipid compartment of surfactant, thereby weakening its overall biophysical activities (43). The primary solvent constituents of ENDS vapor, particularly PG, have emulsifying properties that make them useful solvents in cosmetic and food additives (44). Such emulsifying properties, however, have the potential to initiate structural perturbations in the overall lipid phase of surfactant and produce the disruption in lipoprotein homeostasis that we observe after long-term exposure to ENDS solvents. This, along with the 


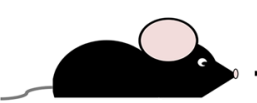

1-month ENDS exposure

A $\mathrm{LDH}$ release $24 \mathrm{~h}$

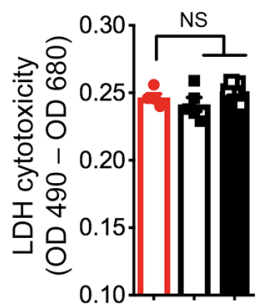

B

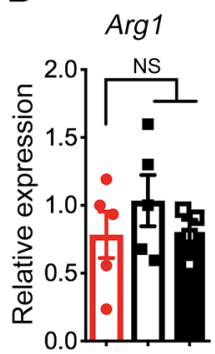

$\mathrm{F} 4 / 80^{+}$cells isolated

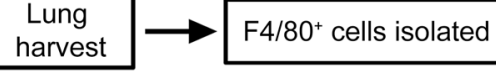

\section{c}

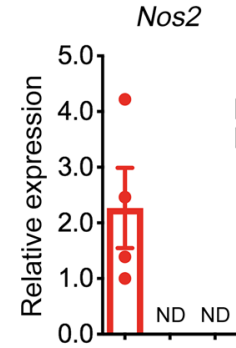

Figure 7. One-month exposure to ENDS vapor attenuates lung-resident macrophage function. Lung-resident $\mathrm{F} 4 / 80^{+}$ macrophages were isolated from whole lung tissue using magnetic beads following 1 month of exposure. Cells were cultured for 24 hours following isolation and supernatants and cells were harvested for the analyses. Individual data points represent technical replicates from pooled lungs of 4 mice per treatment group. (A) Absorbance values following the colorimetric, lactate dehydrogenase (LDH) cytotoxicity assay from 24-hour cultures of Air-, ENDS-vehicle-, and ENDS-nicotine-exposed groups. (B and C) Relative gene expression for Arg1 and Nos2 derived from RNA samples acquired from the cells after 24-hour culture. (D-F) Relative gene expression for (D) cytokines Tnfa and I/1b, (E) costimulatory molecules Cd86 and $C d 80$, and $(\mathbf{F})$ viral recognition receptor T/r7, derived from RNA samples in each treatment group acquired from the cells after 24-hour culture. (G) Relative gene expression for the transcription factor Irf7, a critical factor for type I IFN production, derived from RNA samples acquired from the cells in each treatment group after 24-hour culture. Cells were treated with either polyinosinic:polycytidylic acid (poly I:C) at a concentration of $10 \mu \mathrm{g} / \mathrm{mL}$ or PBS vehicle. All quantified results are expressed as means \pm SEM. $n=4$ or 5 per group. Significance was determined by 1-way ANOVA with Bonferroni's correction for multiple comparisons. ${ }^{* * *} P<0.0001$, ${ }^{*} P<0.01,{ }^{*} P<0.05$. All data shown are representative of 3 or more independent 1-month experiments with $n=4$ or 5 per group. NS, not significant; ND, none detected.

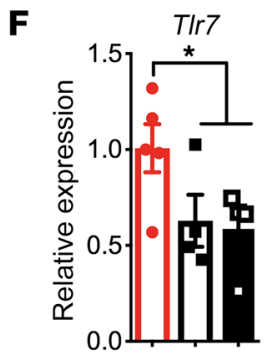

G

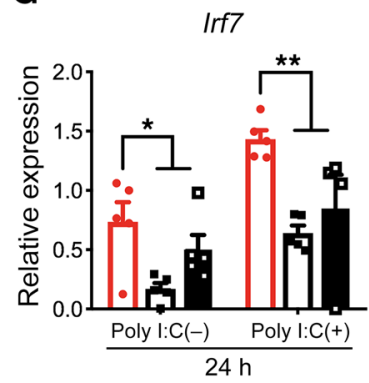

phenotype we observe in our model, raises alarm about the potential deleterious effects that ENDS may have on the alveoli and warrants more systematic evaluation of the pulmonary-specific consequences of ENDS solvents.

Chronic exposure to ENDS in our model has important physiological consequences in innate immunity, as has been observed by other groups in the field $(34,45,46)$. The immunomodulatory effects of nicotine have been well documented in both in vivo and in vitro systems (23). Immune cells, including macrophages, possess nicotinic receptors and binding of nicotine to these receptors reduces inflammatory responses to immune stimuli $(47,48)$. Airway epithelial cells also abundantly express nicotinic receptors, which activate AKT-dependent signaling and modulate surfactant metabolism $(49,50)$. However, we demonstrate that although nicotine may affect cellular responses, PG/VG alone can modulate both pulmonary lipid homeostasis and local innate immune cell function. This corroborates the work of others in the field who have demonstrated molecular changes in human lung tissue in response to vehicle solvents alone $(51,52)$. In response to long-term exposure to ENDS, we reveal a distinct impairment in lung macrophage immune function. Lung-resident macrophages from ENDS-exposed mice exhibit reduced expression of pathogen-sensing proteins (e.g., TLR7) and proteins necessary for induction of critical adaptive immune responses (e.g., CD86, CD80, IL-6) in response to immune stimuli. Concurrently, although ENDS-treated mice lacked lung inflammation under steady states, they responded poorly to acute viral infection with influenza A, exhibiting increased inflammatory infiltrate and severe histopathology late in infection. These observations demonstrate that not only do inhaled aerosols from ENDS disrupt lipid biosynthesis in the lung, they also dampen innate immune mechanisms and increase the susceptibility to inhaled pathogens. Given the extensive number of adolescents using ENDS on a consistent basis, the increased mortality and morbidity demonstrated in our END-exposed infection model should alert officials both at regulatory and epidemiological levels to the potential risk that ENDS use may pose to this vulnerable population.

Although we show aberrant lung macrophage function and lipid alterations, the connections between lipid compositions and functional phenotypes are not well understood and their interrelation can be difficult to unequivocally demonstrate. Changes in membrane lipid composition can broadly affect the organization and activity of signaling proteins in the plasma membrane (53), though specific molecular mechanisms are difficult to specify due to the pleiotropic nature of lipid perturbations. However, it has been shown that tuning membrane composition by dietary lipid 

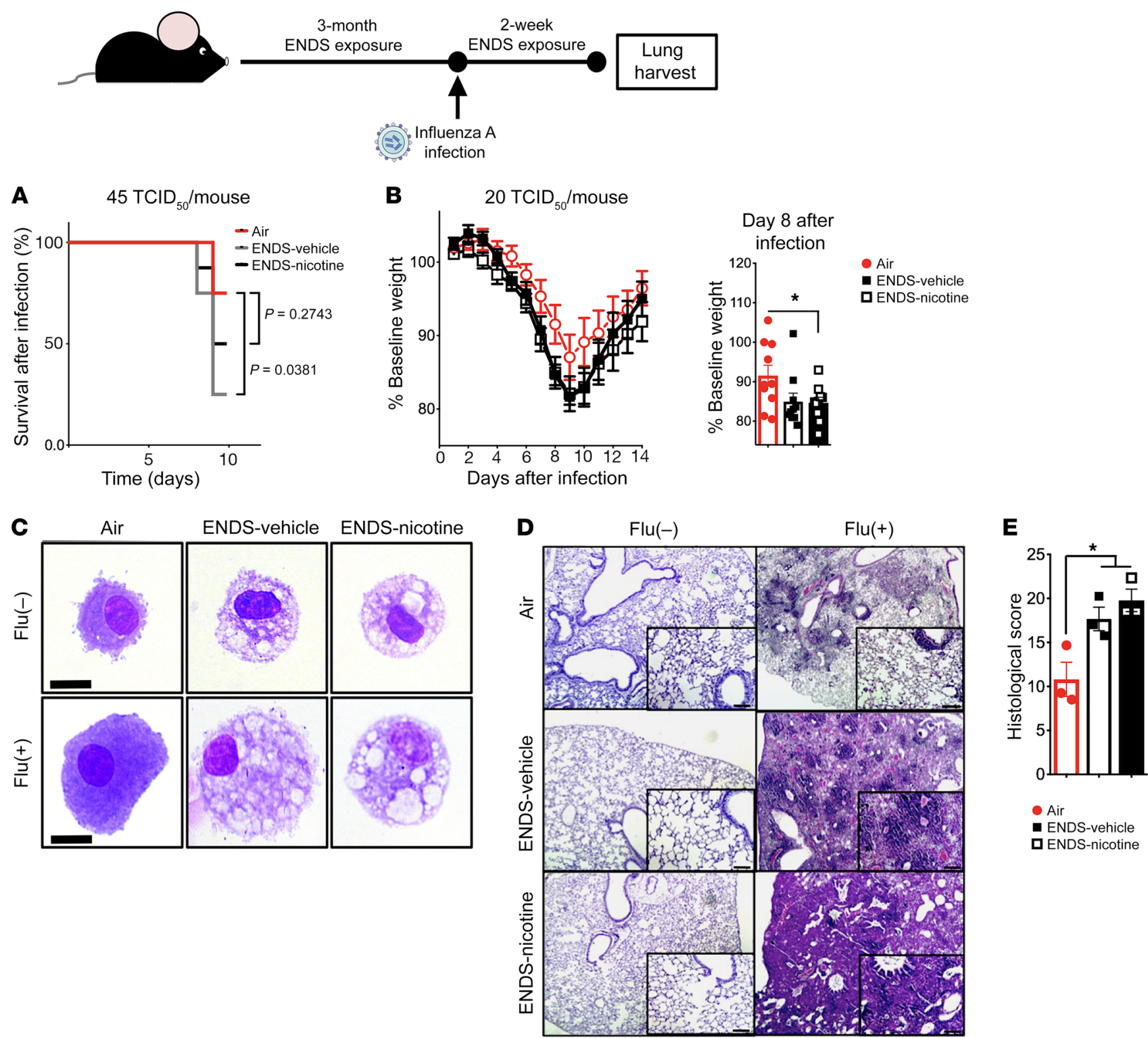

$\mathbf{F}$

IFN- $\gamma$

TNF- $\alpha$

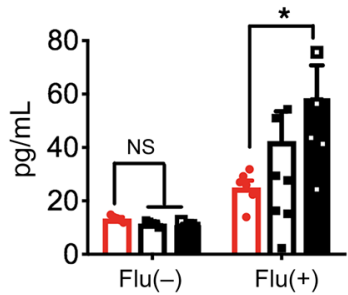

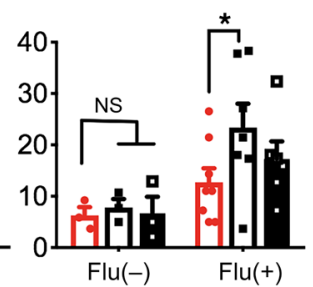

G

G Total lgG (lung)

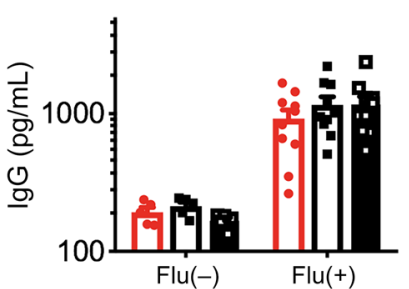

H

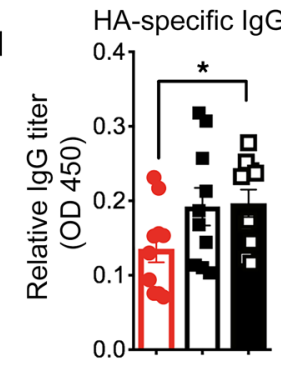

Figure 8. ENDS exposure alters immune responses and recovery from influenza A infection. Eight-week-old mice were exposed to ENDS vapor or room air for 3 months. Subsequently, mice were infected with influenza A (45 TCID $/$ /mouse or $20 \mathrm{TCID}_{50} /$ mouse). (A) Survival curve following infection with highertiter viral infection $-45 \mathrm{TCID}_{50}$ /mouse. $n=8$ per group. Significance was assessed by the log-rank (Mantel-Cox) test. ${ }^{*} P<0.05$. (B) The weight loss and recovery curve following infection with influenza virus $\left(20 \mathrm{TCID}_{50} /\right.$ mouse) with quantification of weight loss on day 8 of infection. Significance was determined by Student's $t$ test. ${ }^{*} P<0.05 ; n=10$ per group. (C) Representative BAL cytospin preparations demonstrating the intracytoplasmic inclusions found in alveolar macrophages irrespective of infection status. Scale bars: $20 \mu \mathrm{m}$. (D) Histological analysis of lung tissue on day 14 following infection. Representative micrographs of H\&E staining. Scale bars: $10 \mu \mathrm{m}$. (E) Objective quantification of pathological changes in lung H\&E micrographs on day 14 following infection. $n=3$ per group. (F) Cytokine concentrations for IFN- $\gamma$ and TNF- $\alpha$ on day 14 following infection as determined by cytokine multiplex arrays. $n=5-9$ per group. Significance was determined by Student's $t$ test. ${ }^{*} P<0.05$. (G and $\left.\mathbf{H}\right)$ Antibody titers for total (G) and hemagglutinin-specific lgC (H) from whole lung homogenate on day 14 following infection as determined by ELISA ( $n=5-10$ per group). Significance was determined by Student's $t$ test or 1-way ANOVA with Bonferroni's correction for multiple comparisons. ${ }^{*} P<0.05$. All data shown are representative of 3 or more independent experiments with $n=5-9$ per group. 


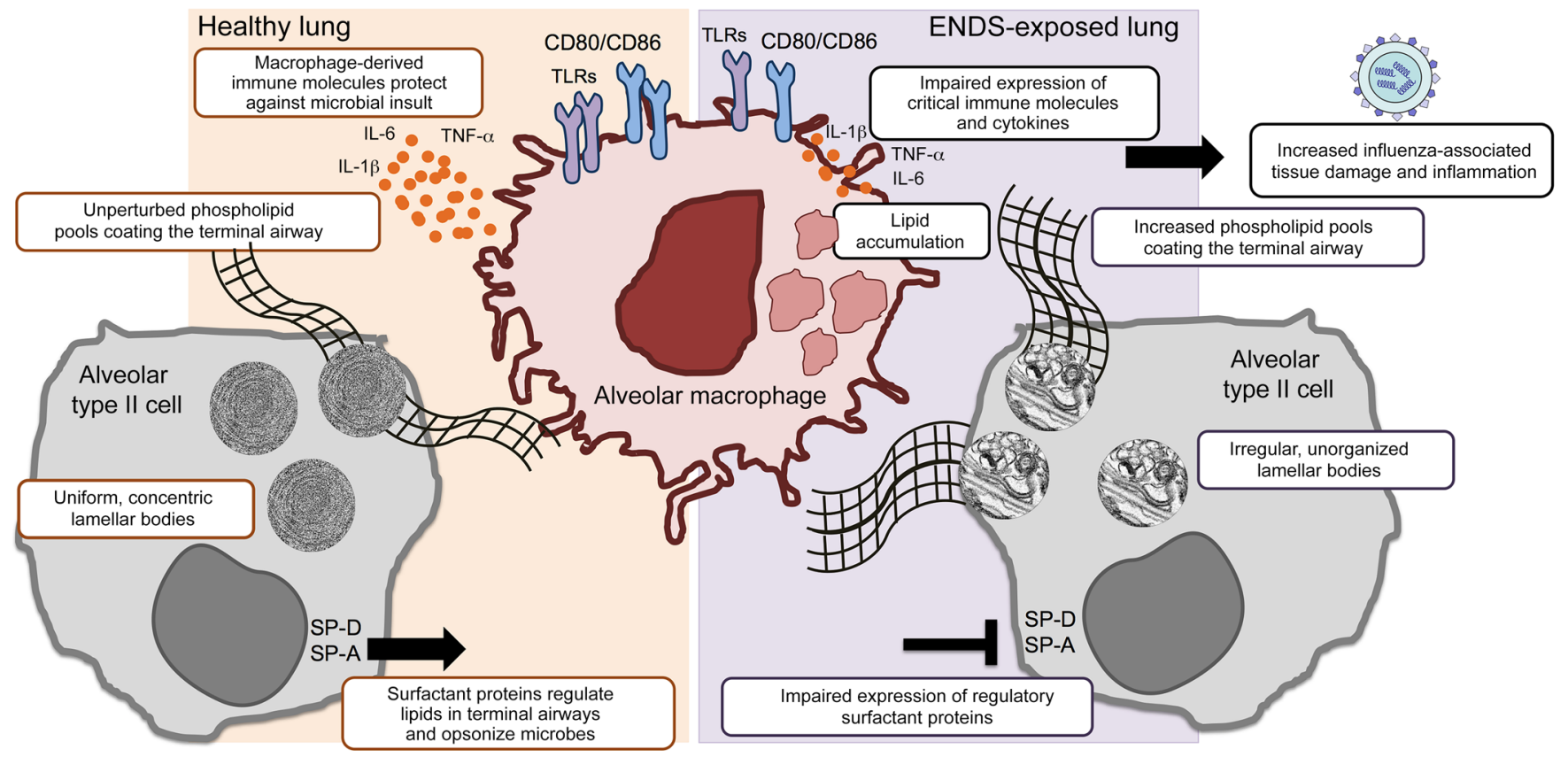

Figure 9. Summary model: ENDS-mediated changes in the lung upon chronic exposure. The lung's delicate surfactant layer is of critical importance to the organ's overall physiology and innate immune function. Both alveolar type II cells and alveolar macrophages are the principal subsets that maintain and catabolize surfactant at the liquid-air interface. Our study reveals that ENDS exposure disrupts both the lipid and protein components of pulmonary surfactant, increasing phospholipid pools in the airway and decreasing the expression of the regulatory surfactant proteins SP-A and SP-D. Lipid deposition and impaired immune function are distinct features of alveolar macrophages upon chronic ENDS treatment. Upon viral infection, ENDS-exposed mice exhibit increased morbidity and mortality with excessive pulmonary damage and inflammation late in infection. Of chief importance, the ENDS-mediated effects observed in our model are independent of the presence of nicotine.

inputs can modulate lineage specification in mesenchymal stem cells (54). The molecular mechanism in this case was related to remodeling of membrane subdomains, which can influence signaling at the plasma membrane. A similar effect was shown by modulating membrane properties using bile acids, which promote the mitogenic signaling downstream of growth factor engagement (55). It is likely that the lipidomic remodeling induced by vapor exposure restructures membrane properties of both surfactant and lung macrophages, potentially perturbing critical biophysical properties, which should be investigated in future studies.

Together, our data highlight the importance of lung lipid balance and overall function of the innate arm of immunity. Further, this study demonstrates an urgent need to better examine the physiological effects of e-cigarette solvents currently in the market. The manufacture and design of e-cigarettes appears to be ever evolving, making it difficult to rigorously and systematically investigate the devices while keeping up with the constant advancements. Although our study investigates a single type of e-cigarette, we uncovered pulmonary effects of vehicle solvent constituents (PG and VG) that are common to a variety of e-cigarette brands. Another important caveat to our study of chronic ENDS exposure is the absence of added flavoring to the ENDS solutions. Among the 7,000 flavors on the market currently, each contains a unique chemical composition capable of enhancing the harmful effects of the vapor components alone. The objective of our study was to investigate how the basic components of ENDS vapor (namely nicotine, PG, and VG) alter the homeostatic function of lipid biosynthesis and immunity in the lungs. Given the results of our study, it is now necessary to further delineate which constituents, PG or glycerol, in ENDS vapor are responsible for our observed findings. Both PG and glycerol are biologically relevant molecules that can serve as substrates for several biochemical reactions, particularly those associated with lipid synthesis. What remains is the determination of the extent to which ENDS solvents and major flavorings disturb the critical lipid balance between alveolar macrophages and ATIIs in the lung of chronic e-cigarette users.

\section{Methods}

Mice. Two-month-old female mice, C57BL/6J background (wild type), were purchased from The Jackson Laboratory. Mice were housed under specific pathogen-free conditions at the Baylor College of Medicine Transgenic Mouse Facility.

ENDS liquid and dosage. We randomly assigned mice to the following groups: Air (room air only), Smoke (traditional tobacco smoke), ENDS-vehicle (PG/VG only), and ENDS-nicotine (PG/VG with $33 \mathrm{mg} /$ $\mathrm{mL}$ nicotine). After random assignment to each treatment group, mice were exposed to their respective conditions for a total of 4 months, unless otherwise specified.

To modify our well-characterized smoking apparatus (27), we used the commercially available Vapor Zeus automatic electronic cigarette from Vapor4Life Inc. The device consists of an automatic battery, which provides 5 volts with a 1300-mAh capacity, and a cartomizer with a 2.5-ohm vertical heating coil and a 6-mL solvent capacity. E-liquids consisted of a $60 \%$ PG and $40 \%$ VG base with either O (ENDS-vehicle) or $33 \mathrm{mg} / \mathrm{mL}$ nicotine (ENDS-nicotine) (Vapor Trading Company). Flavorings were not added to the solvents to permit an unperturbed anal- 
ysis of the effects of the ENDS components (PG/VG and nicotine). We analyzed all e-liquids using a LiChrospher $5-\mu \mathrm{m}$ RP-select B 60- $\AA$, LC column $125 \times 4 \mathrm{~mm}$ on an Agilent 1200 HPLC for nicotine-associated contaminants, as these are often the most common impurities found among e-liquids $(56,57)$. We were unable to detect toxic contaminants, such as anabasine (Supplemental Figure 11).

Mouse model of conventional cigarette smoke and ENDS exposure. Mice were exposed to 4 Marlboro Red 100 cigarettes/day as previously described $(27,32,58,59)$. Briefly, C57BL/6J mice (2-month-old females) were exposed to active smoke from Marlboro cigarettes. Cigarettes were burned at a rate of $4 \mathbf{- 5} \mathrm{min} /$ cigarette to mimic exposure to heated smoke. During each cycle, smoke was forced at a rate $5 \mathrm{~L} /$ min into the exposure chamber intermittently; each smoke cycle is designed to mimic the puffing of actual human smokers and to prevent asphyxiation of the mice. Thus, the smoke cycles provided 3 seconds of heated cigarette smoke interrupted by 20 seconds of air $(5 \mathrm{~L} / \mathrm{min})$ using a time-controlled 2-way valve system (Humphrey). Mice were exposed to 4 cigarettes, 5 days a week for a total of 4 months. We used this number of cigarettes to approximate moderate to heavy smoking habits (e.g., greater than 20 pack-year smoke exposure) in humans.

We compared the volume delivered between independent studies to assess reliability and durability over time and determined that our ENDS delivered consistent volumes between studies (Supplemental Figure 12, A and B). Each cycle of vapor exposure provided 3 seconds of heated e-cigarette vapor, which was interrupted by 20 seconds of $5 \mathrm{~L} / \mathrm{min}$ air using a time-controlled 2-way valve system (Humphrey). The concentration necessary to expose mice with equal amounts of nicotine to that of 4 cigarettes was $33 \mathrm{mg} / \mathrm{mL}$ nicotine for the ENDSnicotine group with a total exposure length of 6 minutes and $25 \mathrm{sec}-$ onds as determined by cotinine ELISA (Abnova) (Supplemental Figure 13). Thus, both ENDS-exposed groups received 4 equal cycles per day, mimicking the 4-cigarette exposure of the Smoke-exposed control.

microCT. Quantification of lung emphysema was determined in collaboration with the Baylor College of Medicine Animal Phenotyping Core using microCT volumetric measurements as described previously (27). Briefly, anesthetized mice were imaged by an animal microCT scanner (Gamma Medica). Images of the chest were then subsequently quantified for emphysema by generating 3-dimensional models of both lungs for volume measurement using Amira 3.1.1 software.

Flow cytometry and intracellular cytokine staining. All flow cytometry was performed on the BD LSRII (BD Biosciences) in collaboration with Baylor College of Medicine's Cytometry and Cell Sorting core, and data were analyzed using FlowJo (Tree Star). RBC-free lung single-cell suspensions were used for flow cytometric analyses of lung antigen-presenting cells. The following mouse-specific antibodies were purchased from BD Pharmingen: BUV395-conjugated anti-CD45 (30-F11), PE/Cy7-conjugated anti-CD11c (HL3), and BV510-conjugated anti-Ly6g (1A8). The following mouse-specific antibody was purchased from eBioscience/Thermo Fisher Scientific: Pacific Blue-conjugated anti-MHCII (M5/114.15.2). The following mouse-specific antibody was purchased from BioLegend: APC/ Cy7-conjugated anti-mouse F4/80 (BM8). Additionally, a UV-excitable and fixable Live/Dead stain (Life Technologies) was implemented for dead cell discrimination.

For intracellular cytokine staining of T cells, lung RBC-free singlecell suspensions were stimulated with $10 \mathrm{ng} / \mathrm{mL}$ phorbol 12-myristate 13-acetate (PMA) (Sigma-Aldrich) and $1 \mu \mathrm{g} / \mathrm{mL}$ ionomycin (Sigma-
Adlrich) in the presence of complete media supplemented with $10 \mu \mathrm{g} /$ $\mathrm{mL}$ Brefeldin A (Sigma-Aldrich) for 6 hours. Cells were then surface stained with mouse-specific antibodies purchased from BD Pharmingen: Pacific Blue-conjugated anti-CD8a (53-6.7), FITC-conjugated anti-CD4 (RM4-5), APC-conjugated anti-CD3 (17A2), and a UVexcitable and fixable Live/Dead stain (Life Technologies). Following the surface stain, cells were fixed with $1 \%$ paraformaldehyde and permeabilized with commercial permeabilization buffer (eBioscience). Intracellular cytokines and transcription factors were then stained using mouse-specific PE/Cy7-conjugated anti-IL-17A (TC11-18H10) and PE-conjugated anti-ROR $\gamma \mathrm{t}(\mathrm{Q} 31-378)$ antibodies.

$m R N A$ isolation and $q P C R$. All cell pellets were treated with QIAzol (Qiagen) and mRNA was extracted using methods described previously (60). All probes, Mmp12 (Mm00500554_m1), Sftpd (Mm00486060_ m1), Sftpa (Mm00499170_m1), Sftpb (Mm00455678_m1), Sftpc (Mm00488144_m1), Atg14 (Mm00553733_m1), Atg7 (Mm00512209_ m1), Becn1 (Mm01265461_m1), Bad (Mm00432042_m1), Bax (Mm00432051_m1), Arg1 (Mm00475988_m1), Nos2 (Mm00440502_ $\mathrm{m} 1), \quad$ Tnfa (Mm00443258_m1), Il1b (Mm00434228_m1), Cd86 (Mm00444540_m1), Cd80 (Mm00711660_m1), Tlr7 (Mm00446590_ $\mathrm{m1})$, Irf7 (Mm00516793_g1), Il6 (Mm00446190_m1), Ifng (Mm01168134_m1), Abca1 (Mm00442646_m1), Abcg1 (Mm00437390_ m1), Abca3 (Mm00550501_m1), Pcyt1a (Mm00447774_m1), Lpcat (Mm00461015_m1), Csf2 (Mm01290062_m1), Spi1 (Mm00488140_ $\mathrm{m} 1$ ), and Csf2ra (Mm00438331_g1) were purchased from Thermo Fisher Scientific. Relative expression was determined using the $\Delta \Delta C T$ method with 18S ribosomal RNA (Hs99999901_s1) as an endogenous control.

$B A L$ fluid differential and lipid staining. Collection of the BAL fluid was conducted as described previously (27). Briefly, BAL fluid was collected by instilling $0.8 \mathrm{~mL}$ of sterile PBS and immediately withdrawing fluid for a total of 2 times. Total cell counts were determined by standard hemocytometer. Subsequently, differential BAL cell counts were assessed by centrifuging $200 \mu \mathrm{L}$ of BAL fluid onto a cytospin slide preparation and quantified using HEMA3 stain (Protocol, Thermo Fisher Scientific). Similarly, Oil Red O staining was performed on paraformaldehyde-fixed, BAL fluid cytospin preparations using $0.05 \%$ Oil Red O in $100 \%$ PG followed by a hematoxylin counterstain as described previously (61).

Antibodies and immunoassays. Single-cell suspensions were obtained from the lungs by homogenizing the tissue through a $45-\mu \mathrm{m}$ cell strainer (BD Falcon). Following centrifugation, supernatants were subsequently harvested for cytokine quantification using a multiplex array (EMD Millipore) for a selected group of cytokines (IL-6, IL-17A, TNF- $\alpha$, IFN- $\gamma$, IL-4, IL-13, IL-10, and IL-13). All chemokine analyses were performed on BAL fluid supernatants using a ProcartaPlex Immunoassay (Thermo Fisher Scientific) for selected chemokines (CCL2, CCL3, CCL4 CCL5, CCL11, CXCL1, and CCL20). For the determination of antibody titers in the influenza study, the aforementioned supernatants were used for IgG-specific ELISAs (Southern Biotechnology) as previously described (32). For pulmonary surfactant-specific ELISAs, specifically SP-D (R\&D Systems), BAL fluid was acquired as described above. Following centrifugation, the supernatants were harvested and used for immunoassays.

Influenza $\mathrm{A}$ infection. Mice were infected with influenza $\mathrm{A} / \mathrm{Hong}$ Kong/8/68 (H3N2) (A/HK/68) following 3-month exposure to air or ENDS using previously described methods (32). Briefly, following 3 months of ENDS exposure, mice were infected with an aerosolized lethal (45 $\mathrm{TCID}_{50} /$ mouse) or sublethal dose (20 TCID 50 /mouse) of A/HK/68. 
The frozen, clinical stock was diluted in $0.05 \%$ gelatin in Eagle's minimal essential medium (Sigma-Aldrich); mice were placed in a sealed chamber and underwent aerosolized viral exposure for 20 minutes. Viral titers were determined before and after aerosolization. Mouse weight loss and recovery were assessed for 10 (lethal dose) or 14 days (sublethal dose) following infection, and the mice were then euthanized.

Histopathological scoring. Hematoxylin- and eosin-stained (H\&Estained) slides from influenza-infected mice were coded and scored as described previously (32). A blinded observer scored the tissue from 0 (absent) to 4 (severe) for the following parameters: interstitial and endothelial inflammation, bronchitis, edema, thrombi, and percentage of lung surface exhibiting diffuse inflammatory infiltrate according to previously published guidelines (62).

BAL fluid lipid quantification. Freshly harvested BAL fluids were separated from cells by centrifugation. Pelleted cells were washed twice with sterile PBS to remove any lipids found in the supernatant. Cells were quantified using a standard hemocytometer as described above and equal numbers of cells were pelleted from each mouse and lysed using $5 \%$ Triton X-100 as instructed by the manufacturer's guidelines. Cellular lipids were quantified using a Glycerol Quantification Kit (BioAssay Systems) and a Phospholipid Quantification Kit (Sigma-Aldrich).

Histological evaluation of lung tissue. Following the exposure period, mice were euthanized and lungs were perfused with $4 \%$ paraformaldehyde via intratracheal cannulation at $20-\mathrm{cmH}_{2} \mathrm{O}$ pressure, as previously described (58). Following the fixation, lung tissue then underwent ethanol dehydration and embedding in paraffin wax. Paraffin-embedded tissue was then sliced into $5-\mu \mathrm{m}$ sections and stained with either H\&E or prepared for immunohistochemical staining.

Mass spectrometry shotgun lipidomics. To extensively profile the lipid species in the BAL fluid, we used a high-resolution TripleTOF 5600 equipped with a TurboVTM ion source (AB Sciex) in collaboration with the Baylor College of Medicine Metabolomics Core according to described previously methods (63). Freshly collected BAL fluid was kept on ice throughout processing, and pooled from 3 separate mice to constitute a single sample for lipidomic analysis. Both acellular fluid and pelleted BAL cells were sent for lipidomic analysis. After the acellular supernatant was removed, cells were washed twice with sterile PBS and quantified. Equal numbers of cells were used for each condition $\left(5 \times 10^{5}\right.$ cells). A sample size of 3 was used for each treatment condition. All samples were stored at $-80^{\circ} \mathrm{C}$ until lipid extraction was performed. Extraction was performed using a 2:2:2 volume ratio of water/methanol/dichloromethane.

TEM. Following euthanasia, mice were exsanguinated to remove all blood from the lungs. The lungs were perfused with a TEM primary fixative (2\% paraformaldehyde, $2.5 \%$ glutaraldehyde, and $2 \mathrm{mM} \mathrm{CaCl}_{2}$ in $0.1 \mathrm{M}$ cacodylate buffer) and the smallest lung lobe was removed for further EM tissue processing. All subsequent processing and mounting of tissue were done in collaboration with the Baylor College of Medicine Integrated Microscopy Core.

Lamellar body quantification. Electron micrographs of ATIIs were first coded. Subsequently, a blinded observer quantified the total number of lamellar bodies as well as the number of irregular lamellar bod- ies per cell. Lamellar bodies were considered irregular if they failed to exhibit the uniform, concentric structure that is characteristic of the specialized organelles.

Macrophage isolation and culture. RBC-free, lung single-cell suspensions were labeled with paramagnetic, bead-conjugated anti-F4/80 and separated using an autoMACS (Miltenyi Biotec) according to the manufacturer's instructions. Cells were then cultured for 24 hours in complete RPMI media with $10 \%$ FBS and cells were harvested for gene expression analyses and quantification of LDH (Thermo Fisher Scientific). For stimulation experiments, isolated cells were cultured with 10 $\mu \mathrm{g} / \mathrm{mL}$ poly I:C (Invivogen) or vehicle (PBS) for 24 hours and cells were harvested for gene expression analyses.

Statistics. All statistical analyses were conducted using GraphPad Prism software. All data are represented as the mean values with error bars representing standard error of the mean (SEM). Comparison of BAL fluid cell numbers, lung volume quantifications, gene expression, cytokine concentration, and lipid concentration between air-exposed or smoke-exposed mice and the ENDS-vehicle and ENDS-nicotine mice were accomplished using 1-way analysis of variance (ANOVA) or Student's $t$ test. We considered $P$ values of 0.05 or lower significant.

Study approval. All experimental protocols used were approved by the Institutional Animal Care and Use Committee of Baylor College of Medicine and the National Research Council Guide for the Care and Use of Laboratory Animals.

\section{Author contributions}

MCM and FK designed and analyzed experiments and wrote the manuscript with input from coauthors. CTL, BHG, HYT, NB, RY, and LZS performed smoke- and ENDS vapor-exposure experiments. MJH and BEG aided in the design of influenza infection experiments. PP designed and helped pilot the ENDS vapor-exposure model. NP, IL, RS, MJC, and DBC designed and analyzed experiments and provided necessary reagents.

\section{Acknowledgments}

This work was supported by NIH grants R01 ES029442-01 and R01 AI135803-01; VA Merit grant CX000104; by the Cytometry and Cell Sorting Core at Baylor College of Medicine with funding from the NIH (AI036211, CA125123, and RR024574); and the expert assistance of Joel M. Sederstrom. This project was also supported by the Metabolomics Core at Baylor College of Medicine under the expert assistance of Nagireddy Putluri and the Integrated Microscopy Core at Baylor College of Medicine with the assistance of Fabio Stossi. This work was supported by CPRIT RP160283 - Baylor College of Medicine Comprehensive Cancer Training Program. The contents of this manuscript do not represent the views of the US Department of Veterans Affairs or the US Government.

Address correspondence to: Farrah Kheradmand, 1 Baylor Plaza mailstop-M915A, Houston, Texas 77030, USA. Phone: 713.798.8622; Email: farrahk@bcm.edu.
1. Regan AK, Promoff G, Dube SR, Arrazola R. Electronic nicotine delivery systems: adult use and awareness of the 'e-cigarette' in the USA. Tob Control. 2013;22(1):19-23.

2. Gentzke AS, et al. Vital signs: Tobacco product use among middle and high school students United States, 2011-2018. MMWR Morb Mortal Wkly Rep. 2019;68(6):157-164.

3. Mirbolouk M, et al. Prevalence and distribution of e-cigarette use among U.S. adults: Behavioral
Risk Factor Surveillance System, 2016. Ann Intern Med. 2018;169(7):429-438.

4. McCarthy M. E-cigarettes are major threat to young people's health, says US surgeon general. BMJ. 2016;355:i6652. 
5. Zhu SH, et al. Four hundred and sixty brands of e-cigarettes and counting: implications for product regulation. Tob Control. 2014;23(Suppl 3):iii3-iii9.

6. Soneji S, et al. Association between initial use of e-cigarettes and subsequent cigarette smoking among adolescents and young adults: a systematic review and meta-analysis. JAMA Pediatr. 2017;171(8):788-797.

7. McCabe SE, Veliz P, McCabe VV, Boyd CJ. Smoking behaviors and intentions among current e-cigarette users, cigarette smokers, and dual users: A national survey of U.S. high school seniors. Prev Med. 2017;99:228-235.

8. Zhong J, Cao S, Gong W, Fei F, Wang M. Electronic cigarettes use and intention to cigarette smoking among never-smoking adolescents and young adults: a meta-analysis. Int J Environ Res Public Health. 2016;13(5):E465.

9. Logue JM, et al. Emissions from electronic cigarettes: assessing vapers' intake of toxic compounds, secondhand exposures, and the associated health impacts. Environ Sci Technol. 2017;51(16):9271-9279.

10. Salamanca JC, Meehan-Atrash J, Vreeke S, Escobedo JO, Peyton DH, Strongin RM. E-cigarettes can emit formaldehyde at high levels under conditions that have been reported to be non-averse to users. Sci Rep. 2018;8(1):7559.

11. Sleiman $\mathrm{M}$, et al. Emissions from electronic cigarettes: key parameters affecting the release of harmful chemicals. Environ Sci Technol. 2016;50(17):9644-9651.

12. Farsalinos KE, Kistler KA, Gillman G, Voudris V. Evaluation of electronic cigarette liquids and aerosol for the presence of selected inhalation toxins. Nicotine Tob Res. 2015;17(2):168-174.

13. Stephens WE. Comparing the cancer potencies of emissions from vapourised nicotine products including e-cigarettes with those of tobacco smoke. Tob Control. 2018;27:10-17.

14. Tommasi S, Bates SE, Behar RZ, Talbot P, Besaratinia $\mathrm{A}$. Limited mutagenicity of electronic cigarettes in mouse or human cells in vitro. Lung Cancer. 2017;112:41-46.

15. Itoh M, Aoshiba K, Herai $\mathrm{Y}$, Nakamura $\mathrm{H}$ Takemura T. Lung injury associated with electronic cigarettes inhalation diagnosed by transbronchial lung biopsy. Respirol Case Rep. 2018;6(1):e00282.

16. McCauley L, Markin C, Hosmer D. An unexpected consequence of electronic cigarette use. Chest. 2012;141(4):1110-1113.

17. Nkadi PO, Merritt TA, Pillers DA. An overview of pulmonary surfactant in the neonate: genetics, metabolism, and the role of surfactant in health and disease. Mol Genet Metab. 2009;97(2):95-101.

18. Griese M. Pulmonary surfactant in health and human lung diseases: state of the art. Eur Respir J. 1999;13(6):1455-1476

19. Veldhuizen EJ, Haagsman HP. Role of pulmonary surfactant components in surface film formation and dynamics. Biochim Biophys Acta. 2000;1467(2):255-270.

20. Wright JR. Immunoregulatory functions of surfactant proteins. Nat Rev Immunol. 2005;5(1):58-68.

21. Miles PR, Ma JY, Bowman L. Degradation of pulmonary surfactant disaturated phosphatidylcholines by alveolar macrophages. J Appl Physiol.
1988;64(6):2474-2481.

22. Trapnell BC, Whitsett JA, Nakata K. Pulmonary alveolar proteinosis. $N$ Engl J Med. 2003;349(26):2527-2539.

23. Kalra R, Singh SP, Pena-Philippides JC, Langley RJ, Razani-Boroujerdi S, Sopori ML. Immunosuppressive and anti-inflammatory effects of nicotine administered by patch in an animal model. Clin Diagn Lab Immunol. 2004;11(3):563-568.

24. Minna JD. Nicotine exposure and bronchial epithelial cell nicotinic acetylcholine receptor expression in the pathogenesis of lung cancer. JClin Invest. 2003;111(1):31-33.

25. US Food and Drug Administration, Department of Health and Human Services. CFR - Code of Federal Regulations Title 21, Volume 3. Revised as of April 1, 2018. https://www.accessdata.fda. gov/scripts/cdrh/cfdocs/cfcfr/cfrsearch.cfm? fr=184.1666; https://www.accessdata.fda.gov/ scripts/cdrh/cfdocs/cfcfr/CFRSearch.cfm? $\mathrm{fr}=182.1320 \&$ SearchTerm=glycerin. Accessed August 27, 2019.

26. Kheradmand F, Shan M, Xu C, Corry DB. Autoimmunity in chronic obstructive pulmonary disease: clinical and experimental evidence. Expert Rev Clin Immunol. 2012;8(3):285-292.

27. Shan M, et al. Cigarette smoke induction of osteopontin (SPP1) mediates $\mathrm{T}(\mathrm{H}) 17$ inflammation in human and experimental emphysema. Sci Transl Med.2012;4(117):117ra9.

28. Bernhard W, et al. Phosphatidylcholine molecular species in lung surfactant: composition in relation to respiratory rate and lung development. Am J Respir Cell Mol Biol. 2001;25(6):725-731.

29. Holm BA, Wang Z, Egan EA, Notter RH. Content of dipalmitoyl phosphatidylcholine in lung surfactant: ramifications for surface activity. Pediatr Res. 1996;39(5):805-811.

30. Shibata Y, Berclaz PY, Chroneos ZC, Yoshida M, Whitsett JA, Trapnell BC. GM-CSF regulates alveolar macrophage differentiation and innate immunity in the lung through PU.1. Immunity. 2001;15(4):557-567.

31. Honda K, et al. IRF-7 is the master regulator of type-I interferon-dependent immune responses. Nature. 2005;434(7034):772-777.

32. Hong MJ, et al. Protective role of $\gamma \delta \mathrm{T}$ cells in cigarette smoke and influenza infection. Mucosal Immunol. 2018;11(3):894-908.

33. Lerner CA, et al. Vapors produced by electronic cigarettes and e-juices with flavorings induce toxicity, oxidative stress, and inflammatory response in lung epithelial cells and in mouse lung. PLoS ONE. 2015;10(2):e0116732.

34. Reidel B, et al. E-cigarette use causes a unique innate immune response in the lung, involving increased neutrophilic activation and altered mucin secretion. Am J Respir Crit Care Med. 2018;197(4):492-501.

35. Crotty Alexander LE, et al. Chronic inhalation of e-cigarette vapor containing nicotine disrupts airway barrier function and induces systemic inflammation and multiorgan fibrosis in mice. Am J Physiol Regul Integr Comp Physiol. 2018;314(6):R834-R847.

36. Hussell T, Bell TJ. Alveolar macrophages: plasticity in a tissue-specific context. Nat Rev Immunol. 2014;14(2):81-93.
37. Gautier EL, et al. Gene-expression profiles and transcriptional regulatory pathways that underlie the identity and diversity of mouse tissue macrophages. Nat Immunol. 2012;13(11):1118-1128.

38. Wright JR, Clements JA. Metabolism and turnover of lung surfactant. Am Rev Respir Dis. 1987;136(2):426-444.

39. Ikegami M, Hull WM, Yoshida M, Wert SE, Whitsett JA. SP-D and GM-CSF regulate surfactant homeostasis via distinct mechanisms. Am J Physiol Lung Cell Mol Physiol. 2001;281(3):L697-L703.

40. Korfhagen TR, et al. Surfactant protein-D regulates surfactant phospholipid homeostasis in vivo. J Biol Chem. 1998;273(43):28438-28443.

41. Fukuzawa $T$, et al. Lung surfactant levels are regulated by Ig-Hepta/GPR116 by monitoring surfactant protein D. PLoS ONE. 2013;8(7):e69451.

42. Ernst R, Ballweg S, Levental I. Cellular mechanisms of physicochemical membrane homeostasis. Curr Opin Cell Biol. 2018;53:44-51.

43. Pérez-Gil J. Structure of pulmonary surfactant membranes and films: the role of proteins and lipid-protein interactions. Biochim Biophys Acta. 2008;1778(7-8):1676-1695.

44. Jacob SE, Scheman A, McGowan MA. Propylene glycol. Dermatitis. 2018;29(1):3-5.

45. Hwang JH, et al. Electronic cigarette inhalation alters innate immunity and airway cytokines while increasing the virulence of colonizing bacteria. J Mol Med. 2016;94(6):667-679.

46. Ween MP, Whittall JJ, Hamon R, Reynolds PN, Hodge SJ. Phagocytosis and inflammation: exploring the effects of the components of e-cigarette vapor on macrophages. Physiol Rep. 2017;5(16):e13370.

47. Kawashima K, Fujii T. The lymphocytic cholinergic system and its biological function. Life Sci. 2003;72(18-19):2101-2109.

48. Gahring LC, Osborne-Hereford AV, Vasquez-Opazo GA, Rogers SW. Tumor necrosis factor alpha enhances nicotinic receptor up-regulation via a p38MAPK-dependent pathway. J Biol Chem. 2008;283(2):693-699.

49. West KA, et al. Rapid Akt activation by nicotine and a tobacco carcinogen modulates the phenotype of normal human airway epithelial cells. JClin Invest. 2003;111(1):81-90.

50. Zou W, et al. Nicotine reduces the levels of surfactant proteins $A$ and $D$ via Wnt $/ \beta$-catenin and PKC signaling in human airway epithelial cells. Respir Physiol Neurobiol. 2016;221:1-10.

51. Ghosh A, et al. Chronic e-cigarette exposure alters the human bronchial epithelial proteome. Am J Respir Crit Care Med. 2018;198(1):67-76.

52. Shen Y, Wolkowicz MJ, Kotova T, Fan L, Timko MP. Transcriptome sequencing reveals e-cigarette vapor and mainstream-smoke from tobacco cigarettes activate different gene expression profiles in human bronchial epithelial cells. Sci Rep. 2016;6:23984.

53. Zhou Y, Hancock JF. Deciphering lipid codes: K-Ras as a paradigm. Traffic. 2018;19(3):157-165.

54 . Levental KR, et al. $\omega-3$ polyunsaturated fatty acids direct differentiation of the membrane phenotype in mesenchymal stem cells to potentiate osteogenesis. Sci Adv. 2017;3(11):eaao1193.

55. Zhou Y, et al. Bile acids modulate signaling by functional perturbation of plasma membrane domains. 
J Biol Chem. 2013;288(50):35660-35670.

56. Famele M, et al. Liquid chromatography with tandem mass spectrometry method for the determination of nicotine and minor tobacco alkaloids in electronic cigarette refill liquids and second-hand generated aerosol. J Sep Sci. 2017;40(5):1049-1056.

57. Etter JF, Zäther E, Svensson S. Analysis of refill liquids for electronic cigarettes. Addiction. 2013;108(9):1671-1679.

58. Shan M, et al. Agonistic induction of PPAR $\gamma$ reverses cigarette smoke-induced emphysema. J Clin Invest. 2014;124(3):1371-1381.

59. Yuan X, et al. Activation of C3a receptor is required in cigarette smoke-mediated emphysema. Mucosal Immunol. 2015;8(4):874-885.

60. Goswami S, et al. Divergent functions for airway epithelial matrix metalloproteinase 7 and retinoic acid in experimental asthma. Nat Immunol. 2009;10(5):496-503.

61. Melo RC, D’Ávila H, Bozza PT, Weller PF. Imaging lipid bodies within leukocytes with different light microscopy techniques. Methods Mol Biol. 2011;689:149-161.

62. Soltzberg J, Frischmann S, van Heeckeren C, Brown N, Caplan A, Bonfield TL. Quantitative microscopy in murine models of lung inflammation. Anal Quant Cytol Histol. 2011;33(5):245-252.

63. Piyarathna DWB, et al. Distinct lipidomic landscapes associated with clinical stages of urothelial cancer of the bladder. Eur Urol Focus. 2018;4(6):907-915. 